\title{
1 Demographic stochasticity and resource autocorrelation control biological invasions in
}

\section{2 heterogeneous landscapes}

Andrea Giometto ${ }^{1,2,3,4, *}$, Florian Altermatt ${ }^{2,5, *}$, Andrea Rinaldo ${ }^{1,6}$

1. Laboratory of Ecohydrology, School of Architecture, Civil and Environmental Engineering, École Polytechnique Fédérale de Lausanne, CH-1015 Lausanne, Switzerland,

2. Eawag, Swiss Federal Institute of Aquatic Science and Technology, Department of Aquatic Ecology, CH-8600 Dübendorf, Switzerland,

3. Department of Physics, Harvard University, US-02138 Cambridge, MA, United States of America,

4. Department of Molecular and Cellular Biology, Harvard University, US-02138 Cambridge, MA, United States of America,

5. Department of Evolutionary Biology and Environmental Studies, University of Zürich, CH-8057 Zürich, Switzerland,

6. Dipartimento di Ingegneria Civile, Edile ed Ambientale, Università di Padova, I-35131 Padua, Italy.

*. Corresponding authors, e-mail: andrea.giometto@gmail.com (ORCID ID: 0000-00020544-6023) and florian.altermatt@eawag.ch (ORCID ID: 0000-0002-4831-6958).

This document is the accepted manuscript version of the following article:

Giometto, A., Altermatt, F., \& Rinaldo, A. (2017). Demographic stochasticity and resource autocorrelation control biological invasions in heterogeneous 1andscapes. oikos, 126(11), 1554-1563. https://doi.org/10.1111/oik.04330 


\section{Abstract}

Mounting theoretical evidence suggests that demographic stochasticity, environmental heterogeneity and biased movement of organisms individually affect the dynamics of biological invasions and range expansions. Studies of species spread in heterogeneous landscapes have traditionally characterized invasion velocities as functions of the mean resource density throughout the landscape, thus neglecting higher-order moments of the spatial resource distribution. Here, we show theoretically that different spatial arrangements of resources lead to different spread velocities even if the mean resource density throughout the landscape is kept constant. Specifically, we find that increasing the resource autocorrelation length causes a reduction in the speed of species spread. The model shows that demographic stochasticity plays a key role in the slowdown, which is strengthened when individuals can actively move towards resources. We then experimentally corroborated the theoretically predicted reduction in propagation speed in microcosm experiments with the protist Euglena gracilis by comparing spread in landscapes with different resource autocorrelation lengths. Our work identifies the resource autocorrelation length as a key modulator and a simple measure of landscape susceptibility to biological invasions, which needs to be considered for predicting invasion dynamics within naturally heterogeneous environmental corridors.

\section{Introduction}

Environmental fluctuations and heterogeneity are ubiquitous in nature and are thought to affect nearly all aspects of ecology, ranging from species coexistence to population synchrony, driving range shifts and potentially causing abrupt biotic change (e.g., With and Crist 1995, With 2002). Local population dynamics in temporally fluctuating environments have been studied extensively in recent years (Gonzalez and Holt 2002, Duncan et al. 2013), mainly with respect to population synchrony (Benton et al. 2001, Fox et al. 2011). Theoretical (Vasseur 2007), experimental (Gonzalez and Holt 2002, Fontaine and Gonzalez 2005, Massie et al. 2015) and field (GarcíaCarreras and Reuman 2011) studies have highlighted the relevance of the temporal autocorrelation structure of environmental fluctuations for ecological processes. 
This is surprising, as most environmental variables are typically positively correlated (Benincà et al. 2011), that is, events that are closer in time or in space are more likely to be similar. Focusing on environmental autocorrelation is also of interest in view of the global shift towards 'bluer' climate variables (i.e., more fluctuating) across most continents (e.g., García-Carreras and Reuman 2011). Whereas the ecological significance of temporal fluctuations of the environment has received much investigation, the implications of environmental heterogeneity on spatial dynamics received surprisingly little attention (With 2002, Melbourne et al. 2007). The effect of spatial heterogeneity of process rates or resource distributions may be especially relevant in the context of biological invasions and range shifts (Hastings et al. 2005, Börger et al. 2008).

Natural environments are almost universally heterogeneous (e.g., Holyoak et al. 2005). Much of the current understanding of species spread, however, is based on theoretical models that consider homogeneous landscapes (i.e., landscapes where vital rates and mobility are uniform in space). Traditionally, the propagation of invasive fronts has been modeled with the FisherKolmogorov equation (Fisher 1937, Kolmogorov et al. 1937). This non-linear reaction-diffusion equation was applied extensively to describe field data (Lubina and Levin 1988, Andow et al. 1990). Stochastic generalizations of the Fisher-Kolmogorov equation showed that demographic stochasticity affects the propagation dynamics in uniform landscapes, causing a reduction in the front propagation speed (Hallatschek and Korolev 2009) and an intrinsic variability of the process across replicated invasions (Giometto et al. 2014). In recent years, progress has been achieved in the theoretical understanding of species spread in more complex, heterogeneous or fluctuating environments (Neubert et al. 2000, Melbourne et al. 2007, Dewhirst and Lutscher 2009, Méndez et al. 2010, Pachepsky and Levine 2011, Fronhofer et al. 2017). For example, the study of front propagation in landscapes whose spatial structure includes a complex topology suggests that the presence of bifurcations along the backbone of the propagation may reduce the invasion speed (Méndez et al. 2003, Méndez et al. 2004, Campos et al. 2006, Bertuzzo et al. 2007). Temporal fluctuations in mean dispersal distances were shown to increase the front propagation velocity (Ellner and Schreiber 2012), while temporally uncorrelated fluctuations in demographic parameters were shown to typically reduce it (Méndez et al. 2011).

Here, we focus on biological invasions in landscapes characterized by temporally invariant but spatially heterogeneous resource distributions, which could, for example, reflect the spatial 
composition and quality of soil, gap dynamics in forests and subsequent light availability for understory plants and their associated herbivore fauna or habitat fragmentation due to human land-use (e.g., With and Crist 1995, With 2002). The investigation of species spread in spatially heterogeneous landscapes has mainly focused on the relationship between the mean invasion speed and the percentage of favorable habitat across the landscape (With and Crist 1995, With 2002, Dewhirst and Lutscher 2009), showing that spread may not occur below minimal thresholds of suitable growth habitat. A limited number of empirical works has measured spread rates in heterogeneous and diverse habitats and compared realized spread distances in patchily distributed sites (Bergelson et al. 1994, Bailey et al. 2000, Williams et al. 2016,) or across landscapes with monotonic gradients (e.g., Fronhofer et al. 2017). Spatially heterogeneous landscapes, however, are not only characterized by the mean percentage of favorable habitat or the mean density of resources, but crucially also by their spatial autocorrelation. Currently, we lack both theoretical and experimental studies investigating the role of such autocorrelation in driving the spread of species.

We manipulated the availability of light as a limiting resource with a spatially heterogeneous distribution. Light is the most important resource for photosynthetically active organims, such as higher plants or algae, and these organisms are not only using light as their key resource for growth, but can also adjust their movement in response of light availability (Giometto et al., 2015). Such directional movement can be active movement (e.g., in flagellated algae) or in directed growth/budding of plant stolones. For most plants, especially in forests, the availability of light is spatially highly heterogeneous, and mostly driven by gap dynamics (REF). Thus, gaps due to tree-fall or anthropogenic activities create a patchy distribution of light as a key resource for understory plants, and all of their associated interacting herbivores and pollinators. We were interested in how the arrangement, specifically the autocorrelation of the light availability, can affect spread dynamics of organisms. While we focus on light as a resource, our theoretical work and paralleled experiments are more generic with respect to the specific resource creating the heterogeneity in habitat quality.

We use light as a resource, which is rarely studied, largely because of its extraordinary flexibility in designing and realizing any experimental pattern of imposed heterogeneity, either in space and/or time. This holds true, in particular, in comparison, say, to the experimental creation 
of patchy or fluctuating resource gradients for which gradient climbing, behavioral modules, and natural search strategies are tightly related (e.g. Hein et al. 2016). We postulate that the nearly perfect experimental reproducibility of the statistical structure of heterogeneous resource fields is a key feature that allows us to mimic almost any real-life ecological setting where fluctuations are a factor.

Here, we theoretically and experimentally show that the invasion speed is affected not only by the mean amount of resources along the landscape (or, analogously, by the percentage of suitable habitat), but also by their spatial autocorrelation structure. We find that environmental heterogeneity and demographic stochasticity jointly affect biological invasions. In particular, we show that it is necessary to include demographic stochasticity in models of spread in order to properly understand biological invasions in spatially heterogeneous environments. Our investigation is organized as follows. We first show theoretically, in two models of invasion at different levels of biological detail, that the speed of species spread decreases when the resource autocorrelation length increases. Second, we verify such a prediction in a microcosm experiment with the flagellated protist Euglena gracilis, by manipulating light intensity profiles along linear landscapes (light is an energy source for E. gracilis, as it has chloroplasts and can photosynthesize). Third, we discuss the contribution of each process included in the model to the propagation of biological invasions.

\section{Methods}

\section{Model}

Species spread in heterogeneous linear landscapes is modeled via a stochastic generalization of the Fisher-Kolmogorov equation including demographic stochasticity (Dornic et al. 2005, Bonachela et al. 2012, Giometto et al. 2014, Villa Martín et al. 2015):

$$
\frac{\partial \rho}{\partial \mathrm{t}}=\mathrm{D} \frac{\partial^{2} \rho}{\partial \mathrm{x}^{2}}+\mathrm{r}(\mathrm{I}) \rho\left[1-\frac{\rho}{\mathrm{K}}\right]+\sigma \sqrt{\rho \eta}
$$
where $\rho(x, t)$ is the density of individuals, $D$ is the diffusion coefficient of the species due to the active movement of individuals, $r$ is the growth rate, $\mathrm{K}$ is the carrying capacity, $\sigma$ is a parameter 
describing the amplitude of demographic stochasticity and $\eta$ is a gaussian, zero-mean white noise (i.e., the noise has correlations $\left\langle\eta(\mathrm{x}, \mathrm{t}) \eta\left(\mathrm{x}^{\prime}, \mathrm{t}^{\prime}\right)\right\rangle=\delta\left(\mathrm{x}-\mathrm{x}^{\prime}\right) \delta\left(\mathrm{t}-\mathrm{t}^{\prime}\right)$, where $\delta$ is the Dirac's delta function). Itô's stochastic calculus is adopted, as appropriate for the demographic noise term (Giometto et al. 2014). The diffusion term in equation (1) assumes random movement of individuals. The growth rate $r_{i}=r_{0} I$ is assumed to be a function of the local amount of resources $\mathrm{I}(\mathrm{x})$, which can assume two values: $\mathrm{I}(\mathrm{x})=1$ or $\mathrm{I}(\mathrm{x})=0$. Landscape heterogeneity is thus embedded in the resource profile $\mathrm{I}(\mathrm{x})$. We studied the dimensionless form of equation (Fehler!

Verweisquelle konnte nicht gefunden werden.), which reads (see Supplementary material Appendix 1):

$\frac{\partial \rho^{\prime}}{\partial \mathrm{t} \prime}=\frac{\partial^{2} \rho^{\prime}}{\partial \mathrm{x}^{\prime 2}}+\chi_{\mathrm{I}} \rho^{\prime}\left[1-\rho^{\prime}\right]+\sigma^{\prime \prime} \sqrt{\rho^{\prime}} \eta$,

where $t^{\prime}=r_{0} t, x^{\prime}=\sqrt{D / r_{0}} x, \rho^{\prime}=\rho / K, \sigma^{\prime \prime}=\frac{\sigma}{\left(K^{2} r D\right)^{1 / 4}}$ and $\chi_{I}\left(x^{\prime}\right)$ is the indicator function of the set of $\mathrm{x}^{\prime}$ for which $\mathrm{I}\left(\mathrm{x}^{\prime}\right)=1$. In the following we drop primes for convenience: one can recover the original dimensions by multiplying $\mathrm{t}$ by $\mathrm{r}_{0}$, $\mathrm{x}$ by $\sqrt{\mathrm{r}_{0} / \mathrm{D}}$ and rescaling $\rho$ and $\sigma$ as indicated above. Numerical integration of stochastic partial differential equations with square root noise terms require ad hoc numerical methods, as standard approaches such as the first-order explicit Euler method inevitably produce unphysical negative values for the density $\rho$ (Dornic et al. 2005). Therefore, equation (Fehler! Verweisquelle konnte nicht gefunden werden.) was integrated with the split-step method proposed in Dornic et al. (2005), see Supplementary material Appendix 1 for details.

We generated landscapes with various resource autocorrelation lengths by imposing $\mathrm{I}(\mathrm{x})$ to be composed of subsequent independent patches of suitable $\left(\mathrm{I}(\mathrm{x})=1\right.$ and $\left.\mathrm{r}=\mathrm{r}_{0}\right)$ or unsuitable $(\mathrm{I}(\mathrm{x})=0$ and $\mathrm{r}=0$ ) habitats (Fig 1A). The length of each patch was drawn from an exponential distribution with rate $\mu$. Therefore, each landscape was a stochastic realization of the so-called telegraph process with rate $\mu$ and autocorrelation length $c_{\mathrm{L}}=1 /(2 \mu)$ (see Supplementary material Appendix 1). The mean extent of suitable and unsuitable patches in such landscapes is $1 / \mu$. Because simulated landscapes were finite, we only accepted landscapes with mean resources equal to $\overline{\mathrm{I}}=1 / \mathrm{L} \int_{0}^{\mathrm{L}} \mathrm{I}(\mathrm{x}) \mathrm{dx}=1 / 2$ and autocorrelation length confined to a narrow window around $1 /(2 \mu)$. Examples of landscapes used in the simulations are shown in Fig. 1A. 
We generated 96 landscapes for each value of resource autocorrelation length $\mathrm{c}_{\mathrm{L}}$ and integrated equation (Fehler! Verweisquelle konnte nicht gefunden werden.) numerically for each landscape and for each value of $\sigma \in\{0.1,0.2,0.4,0.6\}$ (Fig. 1B), with initial density profiles localized at the origin. A reflective boundary condition at the origin may cause the extinction of the whole population, which may happen due to demographic stochasticity. Because our investigation is only concerned with successful invasions (i.e., invasions that survive an initial establishment phase), we fixed the left boundary at $\rho=1$, which thus represents a continuous source of individuals. Such a boundary condition ensures that the population does not go extinct in our numerical investigation. For each numerical integration, we measured the position of the front by fixing a threshold value of the density $(\bar{\varrho}=0.15)$ and recording the furthest point from the origin where the cell density was higher than such value. The mean propagation speed for each value of the resource autocorrelation length was computed by fitting a straight line (least-squares fit) to the mean front position versus time in the asymptotic propagation regime (Fig. 6), before any of the replicated invasions reached the end of the landscape.

The traditional way to compute the invasion speed in the deterministic Fisher-Kolmogorov equation is to analyze the traveling wave solutions of the equation. Such procedure cannot be adopted for equation (Fehler! Verweisquelle konnte nicht gefunden werden.), due to the stochastic noise term. We derived a theoretical approximation to the mean front propagation speed, valid for large autocorrelation length $\mathrm{c}_{\mathrm{L}}$ and $\sigma$ (see Supplementary material Appendix 1, section 2.1.2), by characterizing the mean time taken to cross a patch of unfavorable habitat (where $\mathrm{I}=\mathrm{r}=0$ ) of length $\mathrm{z}$. Such mean time is shown (Supplementary material Appendix 1) to

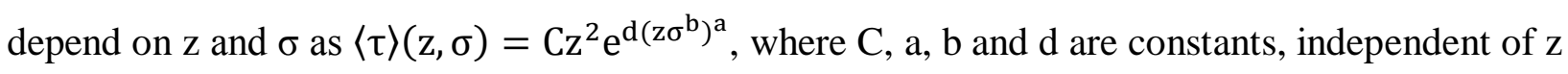
and $\sigma$. Additionally, we characterized the functional dependence of the variance of $\tau$ on $\mathrm{z}$ and $\sigma$ and derived an approximation to the variance of the total time taken by a front to colonize completely a landscape of finite length L (Supplementary material Appendix 1). Our approximation is in good agreement with numerical integrations of equation (Fehler! Verweisquelle konnte nicht gefunden werden.) (Fig. 4).

To test whether deterministic models predict a slowdown of the invading front for increasing resource autocorrelation length, we numerically integrated equation (Fehler! Verweisquelle konnte nicht gefunden werden.) with $\sigma=0$. Additionally, we numerically integrated equation 
193

194

195

196

197

198

199

200

201

202

203

204

205

206

207

208

209

210

211

212

213

214

215

216

217

218

219

220

(Fehler! Verweisquelle konnte nicht gefunden werden.) with $\sigma=0$ imposing a negative growth rate $\mathrm{r}$ in unfavorable patches where $\mathrm{I}=0$ (Supplementary material Appendix 1).

\section{Experiments}

We performed experiments with the flagellated protist Euglena gracilis, acquired from Carolina Biological Supply (NC, USA). A culture of E. gracilis was initialized two weeks prior to the start of the experiment and kept at $22{ }^{\circ} \mathrm{C}$ under constant LED (Light Emitting Diode, model SMD 5050) light of wavelength $469 \mathrm{~nm}$ (emission width approximately $10 \mathrm{~nm}$ ), in a filtered $(0.2 \mu \mathrm{m}$ filter) nutrient medium composed of sterilized spring water and Protozoan Pellets (Carolina Biological Supply, NC, USA) at a density of $0.45 \mathrm{~g} \cdot \mathrm{l}^{-1}$ in a $500 \mathrm{ml}$ Schott flask (for details on the experimental system, see Altermatt et al. 2015). Such a model system has been used successfully in previous experiments to investigate ecological and evolutionary dynamics during range expansions (Giometto et al. 2014, Giometto et al. 2015, Fronhofer and Altermatt 2015, Seymour et al. 2015, Gounand et al. 2017).

In our experiment, light was used as the energy source for $E$. gracilis. To demonstrate that light was crucial for the growth of $E$. gracilis in our experimental setting, we measured $E$. gracilis' growth curves (Fig. 4A) by initializing eight low-density cultures in $10 \mathrm{ml}$ cell culture flasks. Half of such cultures were placed on top of two LEDs (for each culture) operated at a total flux of $1 \mathrm{~mW}$ each. The other half of the cultures were placed on top of two LEDs (for each culture) operated at the same power, but covered with black tape so that no light would penetrate. The spatial arrangement of illuminated and non-illuminated cell culture flasks was randomized.

Light also affects the movement behavior of E. gracilis individuals through a process known as phototaxis, the directed movement of cells towards or away from light (Drescher et al. 2010, Giometto et al. 2015). Specifically, at low to intermediate light intensities, E. gracilis swims towards the light source at a time scale much shorter than the typical generation time. At very high light intensities, negative phototaxis can also be observed, and the plastic reaction of phototaxis can be induced very reliably (Giometto et al. 2015). The light intensity value used in our experiments is smaller than the light intensity value at which negative phototaxis occurs. 
The front propagation experiment was performed in linear landscapes, which were channels drilled on a plexiglass sheet (5 mm wide, $3 \mathrm{~mm}$ deep and $1.9 \mathrm{~m}$ long, respectively, 300, 200 and $10^{5}$ times the size of an individual, see Giometto et al. (2013)), filled with filtered nutrient medium (Fig. 2A). A gasket avoided water spillage and a plexiglass lid was used to seal the system. The experimental replicates were kept in a climatized dark room at $22{ }^{\circ} \mathrm{C}$ for the whole duration of the experiment. Each linear landscape was covered by a black cardboard sheet. Heterogeneous distributions of resources were generated via linear arrays of LEDs (Fig. 2B) controlled via Arduino Uno boards. LEDs in the array were separated by a distance of $\Delta \mathrm{L}=3.12$ $\mathrm{cm}$ from each other and could be switched on or off individually. Switched-on LEDs emitted light with an intensity of $5.2 \mathrm{~W} \cdot \mathrm{m}^{-2}$ within the plexiglass channel, immediately above the LED. The linear landscapes were placed on top of the LED array at a distance of $4.5 \mathrm{~mm}$. The light intensity profile generated by one LED was measured by placing a white paper sheet inside the plexiglass channel and by measuring the irradiance on the sheet with a digital camera operated in grayscale. The total radiant flux of the LED was measured via a calibrated photodiode. Light intensity profiles with the desired autocorrelation length were designed by imposing the probability $\lambda$ of the LED number $i+1$ in the LED array to be switched-on if the LED number $i$ was switched-off, that is, $\mathrm{P}[\mathrm{LED}(\mathrm{i}+1)=\mathrm{ON} \mid \operatorname{LED}(\mathrm{i})=\mathrm{OFF}]=\lambda$. Such Markov Chain was imposed to be symmetric, that is, $\mathrm{P}[\mathrm{LED}(\mathrm{i}+1)=\mathrm{OFF} \mid \mathrm{LED}(\mathrm{i})=\mathrm{ON}]=\lambda$. Small and large values of $\lambda$ generate resource distributions with long and small autocorrelation lengths (approximately equal to $\Delta \mathrm{L} /(2 \lambda)$ ), respectively. Because landscapes were of finite total length, the above procedure could generate by chance resource profiles with autocorrelation length different from the desired one and with a mean frequency of switched-on LEDs different from 1/2. Therefore, the set of resource profiles obtained with the above Markov Chain procedure was restricted to those with a mean frequency of switched-on LEDs equal to $1 / 2$ and in a narrow window of autocorrelation length around the desired one. Therefore, all replicates had the same mean light intensity to $\bar{I}=1 / L \int_{0}^{L} I(x) d x$.

We compared two treatments in the experiment. Treatment 1 consisted of landscapes with identical small autocorrelation length $\left(c_{L} \simeq 2 \mathrm{~cm}\right)$ but different switched-on LED sequences, generated via the Markov Chain procedure with $\lambda=0.75$. Treatment 2 consisted of landscapes with identical large autocorrelation length $\left(c_{L} \simeq 6 \mathrm{~cm}\right)$ but different switched-on LED sequences, generated via the Markov Chain procedure with $\lambda=0.25$. The choice of the large autocorrelation 
length value in the experiment was limited by the total finite length of the experimental setup and was chosen to be less than $1 / 20$ of the total setup length. We initially had six landscape replicates of each treatment, but lost one replicate of Treatment 1 due to leakage. All 11 landscapes had the same total number of switched-on LEDs and the experimental light intensity profiles are shown in Fig. 3. The stated values of autocorrelation length are based on the first-order autocorrelation of the Markov Chain that generated the landscape. The first three LEDs in every landscape were switched-on to allow the local establishment of the inoculated E. gracilis population and to avoid differences between the two treatments in the initial establishment dynamics. Thus, the landscapes generated via the Markov Chain procedure described in the text started at the fourth LED. In Treatment 2 (large autocorrelation length), three landscapes were chosen so that the fourth LED was switched on and the other four were chosen so that the fourth LED was switched off. In other words, the realized Markov Chain started from its stationary distribution. The spatial arrangement of landscapes belonging to the two treatments on the experimental bench was randomized.

At the start of the experiment, we introduced an ensemble of E. gracilis individuals at one end of the linear landscapes. Following the inoculation, we measured for eight consecutive days the density of E. gracilis throughout all replicates by taking pictures with a stereomicroscope (model Olympus SZX16 with the digital camera Olympus DC72) and counting individuals via image analysis (Altermatt et al. 2015).

\section{Statistical analysis}

We used a mixed effect model to compare the speed of the propagating E. gracilis among the two different treatments. Thereby, the autocorrelation treatment was included as a fixed effect, while day and replicate were included as random effect. We repeated this analysis using different choices of threshold values used for determining the front position. The minimum and maximum threshold values employed in the statistical analysis were chosen such that no replicate displayed a retreating front between successive measurements (caused by noise in the density profiles). The test statistics are reported in table 1 for the density threshold value $\bar{\rho}=60 \mathrm{~cm}^{-1}$ and in table 1 for all values of $\rho$ considered. We did not include the first timepoint in the analysis because it was 
measured immediately after the inoculation of E. gracilis in the landscape and thus was identical for all replicates. Because the propagating front reached the end of the landscape at day 4 in some replicates, the front propagation analysis was performed only with the data up to day 3 (included) to avoid spurious border effects due to the finite size of the system.

\section{Model with directed movement towards resources}

Equation (Fehler! Verweisquelle konnte nicht gefunden werden.) does not assume directed movement of individuals towards resources. This directed movement, however, occurs in our experiment and is likely to occur in nature (Andow et al. 1990, Börger et al. 2008, Fronhofer et al. 2017). Additionally, the experimental resource distributions (i.e., the light intensity profiles $I(x)$ ) were not simply sequences of illuminated and non-illuminated spatial patches with sharp edges, but, rather, smooth light intensity profiles alternating between well-lit and dark regions of the landscape according to the spatial arrangement outlined above. Because E. gracilis is capable of detecting light intensity gradients and moving towards well-lit regions of the landscape, this directed movement may affect the invasion dynamics. To assess the net contribution of the directed movement of individuals towards resources, we incorporated in equation (Fehler! Verweisquelle konnte nicht gefunden werden.) the model for phototaxis derived in Giometto et al. (2015). The phototactic term was inferred from measurements of stationary density distributions of $E$. gracilis in the presence of light gradients (such measurements were performed with the same experimental system adopted here) and was shown to reproduce the accumulation dynamics of $E$. gracilis populations accurately in Giometto et al. (2015). The model equation reads:

$$
\frac{\partial \rho}{\partial \mathrm{t}}=\mathrm{D} \frac{\partial^{2} \rho}{\partial \mathrm{x}}+\mathrm{r}(\mathrm{I}) \rho\left[1-\frac{\rho}{\mathrm{K}}\right]+\sigma \sqrt{\rho \eta}
$$

where $\varphi=\mathrm{a}\left(\mathrm{I}-\mathrm{I}_{\mathrm{C}}\right) /\left(\mathrm{I}+\mathrm{I}_{\mathrm{r}}\right)$ is the phototactic potential describing E. gracilis' attraction towards (or against) light (Giometto et al. 2015). The parameters describing $\varphi$ were estimated (Giometto et al. 2015) and were set equal to $\mathrm{a}=1.4 \cdot 10^{8} \mathrm{~m}^{4} \mathrm{~W}^{-1} \mathrm{~s}^{-1}, \mathrm{I}_{\mathrm{r}}=1.7 \mathrm{~W} \mathrm{~m}^{-2}$ and $\mathrm{I}_{\mathrm{C}}=28 \mathrm{~W} \mathrm{~m}^{-2}$. We assumed that $\mathrm{r}$ follows Monod kinetics (the assumption is customary for phytoplankton, Diehl 2002), that is, $r(I)=r_{1} I /\left(I+K_{I}\right)$, where $K_{I}$ is the half-saturation constant. The model (equation 
Fehler! Verweisquelle konnte nicht gefunden werden.) was integrated with parameters suitable to describe the experimental system, $\mathrm{r}_{1}=6 \cdot 10^{-3} \mathrm{~min}^{-1}, \mathrm{~K}_{\mathrm{I}}=1 \mathrm{~W} \mathrm{~m} \mathrm{~m}^{-2}, \mathrm{~K}=300 \mathrm{~cm}^{-1}, \mathrm{D}=0.08 \mathrm{~cm}^{2} \mathrm{~min}^{-1}$ (estimated in Giometto et al. 2015), various values of $\sigma$ (Fig. 8) and initial condition localized at the origin. See Supplementary material Appendix 1 for details on the numerical integration scheme adopted. The slowdown effect caused by the resource autocorrelation structure is also found with other choices of the growth rate dependence on the resource density. In fact, we found that results do not change qualitatively by assuming a linear dependence of $\mathrm{r}$ on $\mathrm{I}$. We used equation (Fehler! Verweisquelle konnte nicht gefunden werden.) to simulate biological invasions in linear landscapes with resource distributions $\mathrm{I}(\mathrm{x})$ exhibiting various autocorrelation lengths. To mimic the experimental setup (Fig. 3), numerical landscapes were generated with the same Markov-chain procedure used to design the experimental landscapes (see Experiment section), where the light intensity profile generated by a single LED (centered in $\mathrm{x}=0$ ) was assumed equal to the best fit of the equation $I(x)=c_{0} /\left(c_{1}^{2}+x^{2}\right)^{2}$ to the measured light intensity profile (see Fig. S1 of Giometto et al. 2015). The total light intensity was kept constant for all landscapes. To further mimic the experiment, we set reflecting boundary conditions for the integration of equation (Fehler! Verweisquelle konnte nicht gefunden werden.) and simulations in which the population went extinct were excluded from the analysis. Therefore, the model equation (Fehler! Verweisquelle konnte nicht gefunden werden.) was specifically derived to reproduce as closely as possible the experimental system at hand. Landscapes used in the simulations were much longer $(18 \mathrm{~m})$ than those used in the experiment in order to avoid border effects. Such numerical settings allowed a clear identification of the invasion front and allowed simulating species spread in landscapes with very large autocorrelation length, which could not be investigated experimentally because of the finite size of the experimental setup.

\section{Results}

Our generalization of the Fisher-Kolmogorov equation (equations Fehler! Verweisquelle konnte nicht gefunden werden. and Fehler! Verweisquelle konnte nicht gefunden werden.) includes demographic stochasticity and resource heterogeneity. Resource heterogeneity affects the spread dynamics through the dependence of the growth rate $r$ on the local amount of resources I 
(Methods). We found that the speed of invasion in the model equation (Fehler! Verweisquelle konnte nicht gefunden werden.) decreases with increasing resource autocorrelation length (Fig. 1B). The mean front propagation speed, in heterogeneous landscapes where resource patch lengths are distributed exponentially with rate $\mu$, depends on $\mathrm{c}_{\mathrm{L}}$ and $\sigma$ asymptotically (i.e., for large $\mathrm{c}_{\mathrm{L}}$ and $\left.\sigma\right)$ as:

$\mathrm{V}=\frac{\mathrm{L}}{\frac{\mu \mathrm{L}}{2} \int_{0}^{\mathrm{L}} \mathrm{dz}\langle\tau\rangle(\mathrm{z}, \sigma) \mu \mathrm{e}^{-\mu \mathrm{z}}} \simeq \frac{8 \mathrm{c}_{\mathrm{L}}^{2}}{\int_{0}^{\infty} \mathrm{dz}\langle\tau\rangle(\mathrm{z}, \sigma) \mathrm{e}^{-\mathrm{z} /\left(2 \mathrm{c}_{\mathrm{L}}\right)}}$

Figs. 1B and 2 show that equation (Fehler! Verweisquelle konnte nicht gefunden werden.) correctly predicts the speed of invasion at large values of $\mathrm{c}_{\mathrm{L}}$ and $\sigma$. In heterogeneous landscapes with different spatial arrangements of favorable and unfavorable patches, if the percentage of space occupied by unfavorable patches is $f_{0} \in(0,1)$ and the distribution of such patches lengths is $\mathrm{p}_{0}(\mathrm{z})$, with mean $\int \mathrm{z} \mathrm{p}_{0}(\mathrm{z}) \mathrm{dz}=1 / \mu$, the asymptotic invasion velocity can be approximated as: $\mathrm{V}=\frac{\mathrm{L}}{\mu \mathrm{f}_{0} \int_{0}^{\infty} \mathrm{dz}\langle\tau\rangle(\mathrm{z}, \sigma) \mathrm{p}(\mathrm{z})}$.

We show in the Supplementary material Appendix 1 that equation (Fehler! Verweisquelle konnte nicht gefunden werden.) correctly predicts the speed of invasion in landscapes with percentages of unfavorable habitat different from $\mathrm{f}_{0}=1 / 2$ (Fig. 5). Note that the speed of invasion according to equations (Fehler! Verweisquelle konnte nicht gefunden werden., Fehler! Verweisquelle konnte nicht gefunden werden.) is a function of the autocorrelation length if the landscapes consist of favorable and unfavorable patches generated through the telegraph process outlined in the Methods section. In general, however, the speed of invasion is not a one-to-one function of the resource autocorrelation length (or of other characteristic length scales of the landscape), but it rather depends on the whole distribution of unfavorable patch lengths through equation (Fehler! Verweisquelle konnte nicht gefunden werden.). The slowdown effect is due to the fact that, in the presence of demographic stochasticity, long patches of unfavorable habitat act as obstacles for the spread of populations. The larger the extent of the unfavorable patch, the longer it takes for a population to cross it. The front propagation speed is also found to be a monotonically decreasing function of the amplitude of demographic stochasticity (Fig. 1B). Accordingly, integrating the model without demographic stochasticity ( $\sigma=0$ in equation Fehler! Verweisquelle konnte nicht gefunden werden., gray dots in Fig. 1B) leads to no discernible 
slowdown of the front in strongly autocorrelated versus weakly autocorrelated landscapes, even when imposing negative values of the growth rate $r$ in unfavorable patches where $\mathrm{I}=0$ (Supplementary material Appendix 1). Such results demonstrate that the local extinctions caused by demographic stochasticity in unfavorable patches are responsible for the observed front slowdown.

Numerical integration of equation (Fehler! Verweisquelle konnte nicht gefunden werden.) shows that the variability of the front position increases for larger values of $c_{L}$ and $\sigma$. This increased variability is caused by two factors: i) two landscapes with identical resource autocorrelation lengths appear increasingly dissimilar for increasing values of the typical patch length $1 / \mu$, ii) the variance of the distribution of waiting times (i.e., the times to cross an unfavorable patch of length z) increases (approximately) quadratically with the mean time $\langle\tau\rangle(\mathrm{z}, \sigma)$ (Fig. 3). These two observations can be used to approximate the fluctuations of the total time spent by the front to colonize a landscape of length L (Fig. 4), as shown in the Supplementary material Appendix 1.

The model (equations Fehler! Verweisquelle konnte nicht gefunden werden. and Fehler! Verweisquelle konnte nicht gefunden werden.) assumes random local movement of individuals. Although such an assumption may be appropriate to describe spread in homogeneous landscapes (Andow et al. 1990, Giometto et al. 2014), individuals might be able to exploit local information on the availability of resources to direct their movement towards more favorable regions (Andow et al. 1990, Fronhofer et al. 2015, Fronhofer et al. 2017). We studied the effect of biased movement towards resources by including an advection term (towards regions endowed with more resources) in equation (Fehler! Verweisquelle konnte nicht gefunden werden.), leading to equation (Fehler! Verweisquelle konnte nicht gefunden werden.). The latter model predicts again that the front propagation speed decreases for increasing resource autocorrelation length, in accordance with the former model (equation Fehler! Verweisquelle konnte nicht gefunden werden.). Integrating equation (Fehler! Verweisquelle konnte nicht gefunden werden.) with and without the advection term shows that the biased local movement towards resources causes an increased slowdown of the invasion front in strongly (compared to weakly) autocorrelated landscapes (Fig. 8). In other words, the biased movement towards resources acts as a spring that keeps the population in favorable patches and works against the exploration of 
unfavorable ones. Excluding demographic stochasticity from the model equation (Fehler! Verweisquelle konnte nicht gefunden werden.) leads again to the elimination of the slowdown effect (inset of Fig. 8).

In the subsequent experiment with $E$. gracilis, we observed a steady front propagation across all landscapes with a mean front propagation speed of $54 \pm 9 \mathrm{~cm} / \mathrm{d}($ mean $\pm \mathrm{SE})$. The mean total number of individuals was 2,420 $\pm 110($ mean \pm SE) at the start of the experiment (day 0 ), $15,000 \pm 800($ mean \pm SE) at the end of the front propagation phase (day 4) and 27,000 $\pm 4,500$ (mean \pm SE) at the end of the experiment (day 8). Thus, the invasion process was a combination of active, directed movement of individuals as well as reproduction. We found a significantly slower front propagation in landscapes in which the resources were strongly spatially autocorrelated (mixed effect model $\mathrm{p}=0.027$, see also Table 1). The result is robust to changes of the threshold value at which the front position is evaluated (Table 1, Figs. 4C and 9). The slowdown effect is visible in Fig. 4C, which shows the mean front position across replicated invasions in the two treatments.

\section{Discussion}

Our theoretical and experimental investigation highlights a yet unrecognized slowdown of biological invasions in heterogeneous landscapes with different resource autocorrelation lengths, which we then corroborated in experimental microcosms specifically designed to single out the effect of resource autocorrelation length on invasion speed. In the experiments, the demographic and movement traits of the study species were fixed and were inherent properties of the species. The accompanying models allowed to single out the individual role and the mutual interconnections of all processes included in the equations to the propagation dynamics in landscapes with different resource autocorrelation lengths.

Our model implicitly assumes that regions of the landscape with positive growth rate $r>0$ can sustain a population of density $K$ indefinitely. One could envision a more general scheme model in which the spatial density of nutrients is fixed and finite at the start of the invasion and declines in time due to uptake by individuals. Investigation of such a model would allow us to test ask whether invasions in heterogeneous landscapes can proceed indefinitely and/or what is the 
probability that a population can overcome unfavorable patches of fixed length before becoming extinct due to the consumption of all the nutrients available in the colonized region of the landscape. In our experiments, we used light as the main energy source, which could be continuously provided at a fixed intensity. A natural analogue of this setting may be found in forest gap dynamics than the low/high light availability in understory plant communities, or in aquatic systems differentially shaded by riparian vegetation. Note, however, that other nutrients in the medium provided the limiting resources which prevented the population from growing indefinitely. Because we did not observe the extinction of our experimental populations, we believe that a generalized model accounting for nutrient depletion behind the front goes beyond the scopes of this investigation, and would not affect our main conclusion.

Our theoretical and experimental study advances our current understanding of the spread of invading organisms in heterogeneous landscapes by addressing the joint effect of spatial environmental autocorrelation and demographic stochasticity on the spread dynamics. As arguably all natural landscapes are characterized by heterogeneous distributions of resources and all populations are subject to demographic stochasticity, our model incorporates two key elements (resource heterogeneity and demographic stochasticity) hitherto often overlooked in the modeling of biological spread.

A major result of our work is that demographic stochasticity is a key factor in the slowdown of front propagation in heterogeneous landscapes. Our finding highlights the importance of including demographic stochasticity in theoretical models because of the many facets through which it affects species spread (Hallatschek and Korolev 2009, Giometto et al. 2014) and ecological dynamics in heterogeneous landscapes (Villa Martín et al. 2015). The implications of the above results challenge the standard approach as stochastic effects are neglected by deterministic, Fisher-Kolmogorov-like models. Because the slowdown effect is only observed when demographic stochasticity is included in the models, our theoretical investigation suggests that the stochastic birth-and-death dynamics are the main drivers of the observed reduction in propagation speed, rather than the movement behavior of individuals in heterogeneous landscapes that has received so far most attention in the literature (Van Dyck and Baguette 2005, Börger et al. 2008). 
Previous studies have investigated the minimum percentage of suitable habitat that allows invasions to spread (With and Crist 1995, With 2002, Dewhirst and Lutscher 2009), suggesting that invasions cannot propagate in landscapes with mean resource density below a critical threshold. Our work shows, complementarily, that the spatial arrangement of resources affects species spread even if the total amount of available resources is kept constant. Thus, it is not only the mean resource density that matters for the front propagation dynamics, because the autocorrelation structure of landscape heterogeneity alone also affects species spread. In general, the larger the patch of unfavorable landscape, the longer it will take for the invading front to overcome it. Such a longer time is caused by the fact that a population cannot have net growth (on average) within an unfavorable patch, and is prone to local extinction due to demographic stochasticity. For large values of autocorrelation length, the mean speed of invasion is mainly determined by the time spent by the front trying to overcome long stretches of unfavorable landscape. In this limit, the mean speed of invasion is mainly determined by the distribution of unfavorable patches lengths, as captured by equation (4).

Our investigation also extends previous works that addressed the effect of temporal environmental fluctuations on species spread (Méndez et al. 2011, Ellner and Schreiber 2012) by showing that the autocorrelation length of the resource distribution should be added to the environmental factors that can slow species spread, along with temporal fluctuations of vital rates (Neubert et al. 2000, Ellner and Schreiber 2012), geometrical heterogeneities of the substrate (Méndez et al. 2003, Méndez et al. 2004, Bertuzzo et al. 2007) and demographic stochasticity (Hallatschek and Korolev 2009). We note that our model does not account for Allee effects that may characterize the growth dynamics of a natural population, for example due to mate limitation. We anticipate that strong Allee effects (i.e., a negative growth rate at small population densities) might cause a reduction in the front propagation speed analogous to the one investigated here, caused by demographic stochasticity. In fact, the origin of such a slowdown effect lies in the local extinction of low-density populations that are able to diffuse through long stretches of unfavorable landscape. Such an extinction may be caused by either demographic stochasticity or by a strong Allee effect. Because our study species does not exhibit a noticeable Allee effect, we have not investigated its dynamics in this investigation. 
The theoretical and experimental results reported here are in strong support and match to empirical real-world examples of invasions. For example, Bergelson et al. (1994) performed a field study with the invading weed Senecio vulgaris and found that the average spatial distance between two generations along linear transects increased when favorable patches were uniformly distributed in space (in the parlance of our work, the transect featured a small autocorrelation length), compared to transects with clumped patches (i.e., endowed with large autocorrelation length). Similar work exists by Bailey et al., (2000) on the spread of the fungal pathogen Rhizctonia solani and provides a complementing view to our investigation by evaluating the effect of the inter-distance between favorable patches on the spread and identifying experimentally the existence of a percolation threshold at a critical level of inter-patch distance. In the framework addressed here, the analog of such percolation threshold corresponds to an autocorrelation length much larger than the average distance traveled by the front during one generation. However, both of these studies also strongly differ from our work. Most importantly, biased active movement towards favorable patches was present in the experiment performed here and embedded in equation (Fehler! Verweisquelle konnte nicht gefunden werden.), while passive dispersal was implemented in Bergelson et al. (1994). Both Bailey et al. (2000) and Bergelson et al. (1994) differ from our study because the landscape and the distribution of resources herein are continuous, whereas they adopted discrete spatial distributions of favorable patches. Although such discrete distributions might provide a good approximation to some fragmented landscapes, continuous heterogeneous distributions may be equally likely to occur in nature.

Our results have important implications for species spread in natural environments, which are generally characterized by resources (seen as any field controlling vital rates, especially reproductive ones) being heterogeneously distributed. Specifically, we here provide a general theoretical framework to interpret the dynamical processes underlying the realized invasions. The typical autocorrelation length of the resource distribution can be inferred from environmental data (Urban et al. 2008) and can be used as a concise indicator for the propagation success of a species of interest. Furthermore, the spatial availability of resources is often altered by human activities, reinforcing the fragmentation of landscapes. In fact, habitat fragmentation may decrease significantly the autocorrelation length of the landscape through the introduction of qualitatively 
512 different patches in the natural environment (With and Crist 1995, With 2002, Holyoak et al.

513 2005). Our results give quantitative grounds to field observations on the effect of environmental

514 heterogeneity on species spread. For instance, Lubina and Levin (1988) observed pauses in the

515

516 spread of the California sea otter (Enhydra lutris) in the presence of habitat discontinuities. Such pauses and the corresponding piecewise-linear propagation of the front (see Fig. 2 of Lubina and Levin 1988) are also found in our model (Fig. 7), which enables to relate the mean spatial extent of habitat discontinuities to the average speed of invasion through equations (Fehler! Verweisquelle konnte nicht gefunden werden.) and (Fehler! Verweisquelle konnte nicht gefunden werden.). An alternation between phases of halt and spread was also found in the range expansion of the cane toad (Chaunus marinus) in Australia (Fig. 2 of Urban et al. 2008). Urban et al. (2008) performed an in-depth analysis of the effect of environmental heterogeneity on the spread of the cane toad in the field and found a statistically significant effect of environmental heterogeneity and, most importantly, of the spatial autocorrelation of environmental variables on the realized patterns of invasion speed. They found such effect in nature in a realized (not replicable) invasion, and thus they could only correlate the realized spread dynamics and its reduction with the landscape autocorrelation. Here, we have given a mathematical framework and an experimental proof showing that the slowdown effect caused by the spatial autocorrelation structure of the landscape is not an artifact of the mathematical model.

\section{Conclusion}

In conclusion, our work demonstrates the need to account for the intrinsic stochasticity of population dynamics to broaden our understanding of ecological processes occurring in spatially extended natural landscapes, which typically display various degrees of heterogeneity. Further work should be dedicated to the modeling and experimentation of species spread in temporally varying landscapes and, possibly, spatially heterogeneous landscapes that fluctuate in time. Drawing from the literature on population dynamics in temporally fluctuating environments, understanding the causal link between the autocorrelation structure of fluctuations and the dynamics of species spread is a promising direction for future research in this area. 
542 We thank Enrico Bertuzzo, Francesco Carrara, Lorenzo Mari and Amos Maritan for many useful

543 discussions. We gratefully acknowledge the support by Swiss Federal Institute of Aquatic Science

544 and Technology (Eawag) discretionary funds and Swiss National Science Foundation Projects P2ELP2_168498, 200021_157174 and PP00P3_150698.

\section{References}

548 Altermatt, F. et al. 2015. Big answers from small worlds: a user's guide for protist microcosms as a model system in ecology and evolution. - Methods Ecol. Evol. 6: 218-231.

Andow, D. A. et al. 1990. Spread of invading organisms. - Landscape Ecol. 4: 177-188.

Bailey, D. J. et al. 2000. Saprotrophic invasion by the soil-borne fungal plant pathogen

Rhizoctonia solani and percolation thresholds. - New Phytol. 146: 535-544.

Benincà, E. et al. 2011. Resonance of plankton communities with temperature fluctuations. - Am. Nat. 178: E85-E95.

555 Benton, T. G. et al. 2001. Population synchrony and environmental variation: an experimental demonstration. - Ecol. Lett. 4: 236-243.

Bergelson, J. et al. 1994. Rates of Weed Spread in Spatially Heterogeneous Environments. -

558 Ecology 74: 999-1011.

559 Bertuzzo, E. et al. 2007. River networks and ecological corridors: Reactive transport on fractals, 560 migration fronts, hydrochory. - Water Resour. Res. 43: W04419.

561 Bonachela, J. et al. 2012. Patchiness and demographic noise in three ecological examples. - J.

562 Stat. Phys. 148: 723-739.

563 Börger, L. et al. 2008. Are there general mechanisms of animal home range behaviour? A review 564 and prospects for future research. - Ecology Letters 11(6), 637-650.

565 Campos, D. et al. 2006. Transport on fractal river networks: application to migration fronts. 566 Theor. Popul. Biol. 69: 88-93. 
567

568

569

570

571

572

573

574

575

576

577

578

579

580

581

582

583

584

585

586

587

588

589

590

591

592

Dewhirst, S. and Lutscher, F. 2009. Dispersal in heterogeneous habitats: thresholds, spatial scales, and approximate rates of spread. - Ecology 90(5): 1338-1345.

Diehl, S. 2002. Phytoplankton, light, and nutrients in a gradient of mixing depths: theory. Ecology 83: 386-398.

Dornic, I. et al. 2005. Integration of Langevin equations with multiplicative noise and the viability of field theories for absorbing phase transitions. - Phys. Rev. Lett. 94: 100601.

Drescher, K. et al. 2010. Fidelity of adaptive phototaxis. - Proc. Natl. Acad. Sci. U.S.A. 107(25): $11171-11176$.

Duncan, A. B. et al. 2013. Stochastic environmental fluctuations drive epidemiology in experimental host-parasite metapopulations. - Proc. R. Soc. London Ser. B 280: 20131747.

Ellner, S. P. and Schreiber, S. J. 2012. Temporally variable dispersal and demography can accelerate the spread of invading species. - Theoretical Population Biology 82(4): 283-298.

Fisher, R. A. 1937. The wave of advance of advantageous genes. - Ann. Hum. Genet. 7: 355-369.

Fontaine, C. and Gonzalez, A. 2005. Population synchrony induced by resource fluctuations and dispersal in an aquatic microcosm. - Ecology 86: 1463-1471.

Fox, J. W. et al. 2011. Phase locking, the Moran effect and distance decay of synchrony: experimental tests in a model system. - Ecol. Lett. 14: 163-168.

Fronhofer, E. A. and Altermatt, F. 2015. Eco-evolutionary feedbacks during experimental range expansions. - Nat. Commun. 6: 6844.

Fronhofer, E. A. et al. 2015. Condition-dependent movement and dispersal in experimental metacommunities. - Ecol. Lett. 18: 954-963.

Fronhofer, E. A. et al. 2017. Information use shapes the dynamics of range expansions into environmental gradients. - Global Ecology and Biogeography 26: 400-411.

García-Carreras, B. and Reuman, D. C. 2011. An empirical link between the spectral colour of climate and the spectral colour of field populations in the context of climate change. - J. Anim. Ecol. 80: 1042-1048. 
593 Giometto, A. et al. 2013. Scaling body size fluctuations. - Proc. Natl. Acad. Sci. U.S.A. 110: $594 \quad 4646-4650$.

595 Giometto, A. et al. 2014. Emerging predictable features of replicated biological invasion fronts. 596 Proc. Natl. Acad. Sci. U.S.A. 111: 297-301.

597 Giometto, A. et al. 2015. Generalized receptor law governs phototaxis in the phytoplankton 598 Euglena gracilis. - Proc. Natl. Acad. Sci. U.S.A. 112: 7045-7050.

599 Gonzalez, A. and Holt, R. D. 2002. The inflationary effects of environmental fluctuations in 600 source-sink systems. - Proc. Natl. Acad. Sci. U.S.A. 99: 14872-14877.

601 Gounand, I. et al. 2017. Subsidies mediate interactions between communities across space. 602 Oikos. In press.

603 Hallatschek, O. and Korolev, K. 2009. Fisher waves in the strong noise limit. - Phys. Rev. Lett. 604 103: 108103.

605 Hastings, A. et al. 2005. The spatial spread of invasions: new developments in theory and 606 evidence. - Ecol. Lett. 8: 91-101.

607 Holyoak, M. et al. 2005. Metacommunities: Spatial Dynamics and Ecological Communities. 608 The University of Chicago Press.

609 Kolmogorov, A. N. et al. 1937. A study of the diffusion equation with increase in the amount of 610 substance, and its application to a biological problem. - Moscow University Mathematics 611 Mechanics Bulletin 1: 1-25.

612 Lubina, J. A. and Levin, S. A. 1988. The Spread of a Reinvading Species: Range Expansion in the 613 California Sea Otter. - Am. Nat. 131: 526-543.

614 Massie, T. M. et al. 2015. Enhanced Moran effect by spatial variation in environmental 615 autocorrelation. - Nat. Commun. 6: 5993.

616 Melbourne, B. A. et al. 2007. Invasion in a heterogeneous world: resistance, coexistence or 617 hostile takeover? - Ecol. Lett. 10: 77-94.

618 Méndez, V. et al. 2003. Speed of reaction-diffusion fronts in spatially heterogeneous media. 619 Phys. Rev. E 68: 041105. 
620 Méndez, V. et al. 2004. Dynamical features of reaction-diffusion fronts in fractals. - Phys. Rev. E 621 69: 016613.

622 Méndez, V. et al. 2010. Reaction-Transport Systems. - Springer.

623 Méndez, V. et al. 2011. Effect of environmental fluctuations on invasion fronts. - J. Theor. Biol. 624 281:31-38.

625 Neubert, M. G. et al. 2000. Invasion speeds in fluctuating environments. - Proc. R. Soc. London 626 Ser. B 267(1453): 1603-1610.

627 Pachepsky, E. and Levine, J. M. 2011. Density Dependence Slows Invader Spread in Fragmented 628 Landscapes. - Am. Nat. 177: 18-28.

629 Seymour, M. et al. 2015. Dendritic network structure and dispersal affect temporal dynamics of 630 diversity and species persistence. - Oikos 124(7): 908-916.

631 Skellam, J. G. 1951. Random dispersal in theoretical populations. - Biometrika 38: 196-218.

632 Urban, M. C. et al. 2008. A toad more traveled: the heterogeneous invasion dynamics of cane 633 toads in Australia. - Am. Nat. 171(3): E134-E148.

634 Van Dyck, H. and Baguette, M. 2005. Dispersal behavior in fragmented landscapes: routine or 635 special movements. - Basic and Appl. Ecol. 6: 535-545.

636 Vasseur, D. A. 2007. Environmental colour intensifies the Moran effect when population 637 dynamics are spatially heterogeneous. - Oikos 116: 1726-1736.

638 Villa Martín, P. et al. 2015. Eluding catastrophic shifts. - Proc. Natl. Acad. Sci. U.S.A. 112(15): 639 E1828-E1836.

640 Williams, J. L. et al. 2016. Rapid evolution accelerates plant population spread in fragmented 641 experimental landscapes. - Science 353(6298): 1-5.

642 Williams, J. L. et al. 2016. The Influence of Evolution on Population Spread through Patchy 643 Landscapes. - The American Naturalist 188(1): 15-26.

644 With, K. A. 2002. The Landscape Ecology of invasive spread. - Conserv. Biol. 16(5): 11926451203. 
646 With, K. A. and Crist, T. O. 1995. Critical Thresholds in Species' Responses to Landscape 647 Structure. - Ecology 76(8): 2446-2459.

648 
649 Tables

650 Table 1: Mixed-effect test statistics.

651

\begin{tabular}{l|l|l|l|l|l}
\multicolumn{2}{l}{ Value } & Std. Error & df & t-value & $p$-value \\
\hline Intercept & 45.98 & 3.27 & 44 & 14.04 & $\mathrm{p}<10^{-4}$ \\
Autocorrelation length & -11.61 & 4.43 & 9 & -2.62 & 0.0279
\end{tabular}

652 Mixed-effect test statistics testing the speed of front propagation, with the autocorrelation length

653 treatment as single fixed effect and time/replicate as random effect. The treatment with small

654 autocorrelation length had 5 replicates, the treatment with large autocorrelation length had 6

655 replicates. The front position was measured at the density threshold value $\bar{\rho}=60 \mathrm{~cm}^{-1}$.

656 

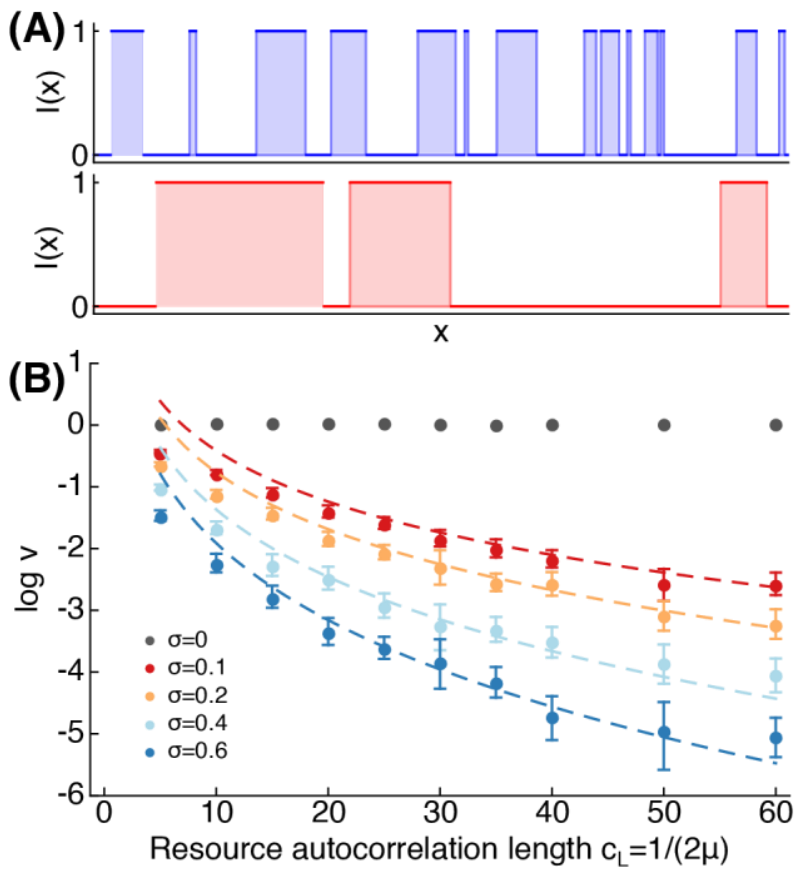

658

659 Figure 1: Mean front propagation in the model (dimensionless equation Fehler! Verweisquelle

660 konnte nicht gefunden werden.). (A) Examples of landscapes with different resource

661 autocorrelation length $c_{L}$, generated via the telegraph process with rate $\mu$ (Methods). (B) The

662 mean invasion speed computed in numerical integrations of the model (equation Fehler!

663 Verweisquelle konnte nicht gefunden werden.) decreases with increasing resource

664 autocorrelation length $c_{\mathrm{L}}$ (log-linear plot) for $\sigma>0$ and is a decreasing function of the amplitude of

665 demographic stochasticity $\sigma$ (different colors according to legend). With $\sigma=0$ the dynamics is

666 deterministic and the mean front propagation speed does not decrease with $\mathrm{z}$ (gray dots). Error

667 bars display the $95 \%$ confidence interval for $\log (\mathrm{v})$, computed with $2 \cdot 10^{3}$ bootstrap samples. Error

668 bars for $\sigma=0$ are smaller than symbols. Dashed lines show the mean front propagation speed

669 computed according to the theoretical approximation (equation Fehler! Verweisquelle konnte

670 nicht gefunden werden.). 


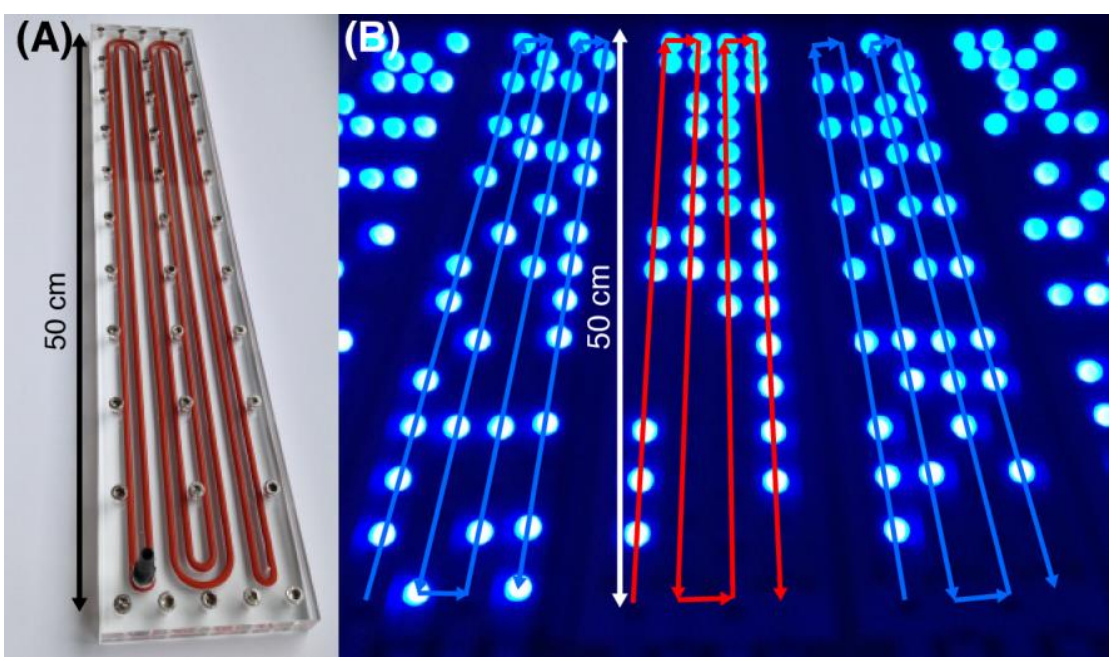

672 Figure 2: Experimental setup. (A) Linear landscapes used in the experiments were channels

673 drilled on a plexiglass sheet. A gasket (orange rubber band) avoided water spillage. (B)

674 Photograph of the LED strips used to control the distribution of resources for E. gracilis. The red 675 and blue lines show the paths of landscapes with large and small resource autocorrelation length, 676 respectively.

677 
678
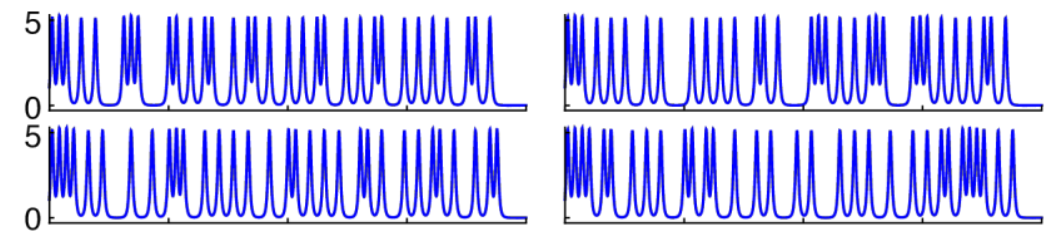

679

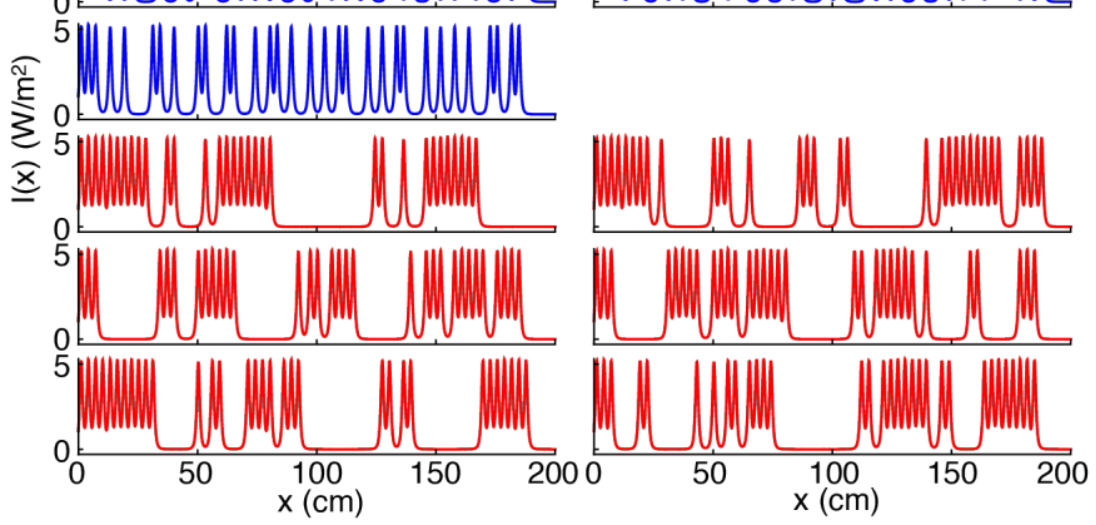

680 Figure 3: Light intensity profiles used in the experiment (Methods). One spread experiment was

681 performed for each landscape. The total light intensity is the same for each landscape. Landscapes

682 with the same color have identical small (blue) or large (red) autocorrelation length of the

683 resource distribution $\mathrm{I}(\mathrm{x})$, but different LED on-off sequences. 

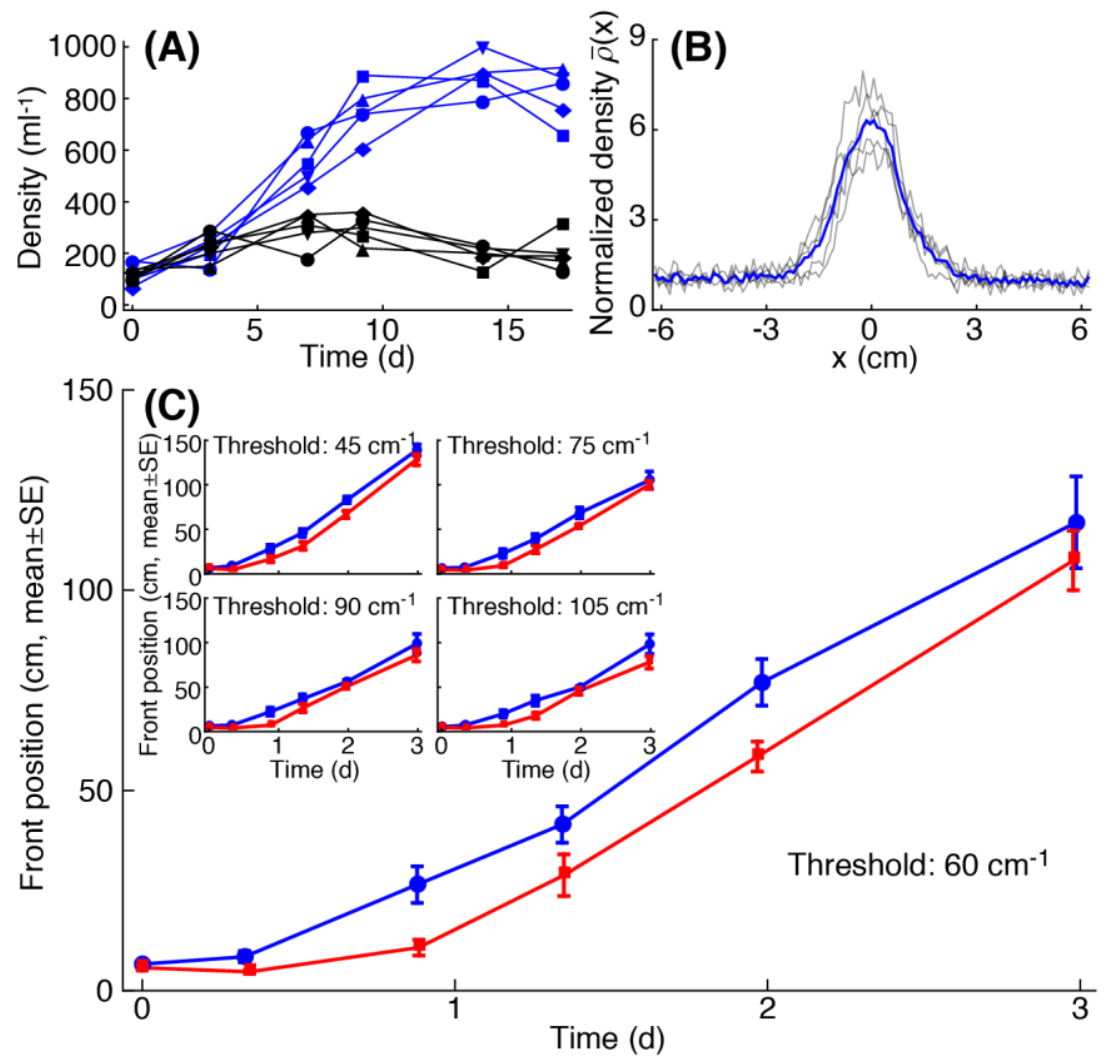

684

Figure 4: Experimental spread in autocorrelated landscapes. (A) Light was used as energy resource for E. gracilis. Replicated measured growth curves show that E. gracilis grows in the presence of light (blue symbols and lines) and does not grow in its absence (black symbols and lines). (B) Replicated measurements (gray lines) of E. gracilis density profiles (normalized by the value at the edge of the imaging window) in the presence of a LED at $\mathrm{x}=0 \mathrm{~cm}$ show that $E$.

690 gracilis populations accumulate around light sources through phototaxis. The blue line denotes the mean density profile across replicates (panel B is redrawn from Giometto et al. 2015). (C)

692 Mean $( \pm$ SE) position of the front, calculated among replicates with identical large (red) or small 693 (blue) resource autocorrelation length at the threshold density value $\bar{\rho}=60 \mathrm{~cm}^{-1}$. The inset shows 694 mean front positions calculated at different threshold density values $\bar{\rho}$ as indicated. The slowdown 695 effect is significant with all choices of $\bar{\rho}$, see Table 1.

696 
698

Demographic stochasticity and resource autocorrelation control biological invasions in heterogeneous landscapes

701

702

Andrea Giometto ${ }^{1,2,3,4, *}$, Florian Altermatt ${ }^{2,5, *}$, Andrea Rinaldo ${ }^{1,6}$

703

704

705

1. Laboratory of Ecohydrology, School of Architecture, Civil and Environmental

706

707

708

709

710

711

712

713

714

715

716

717

718 Engineering, École Polytechnique Fédérale de Lausanne, CH-1015 Lausanne, Switzerland,

2. Eawag, Swiss Federal Institute of Aquatic Science and Technology, Department of Aquatic Ecology, CH-8600 Dübendorf, Switzerland,

3. Department of Physics, Harvard University, US-02138 Cambridge, MA, United States of America,

4. Department of Molecular and Cellular Biology, Harvard University, US-02138 Cambridge, MA, United States of America,

5. Department of Evolutionary Biology and Environmental Studies, University of Zürich, CH-8057 Zürich, Switzerland,

6. Dipartimento di Ingegneria Civile, Edile ed Ambientale, Università di Padova, I-35131 Padua, Italy.

*. Corresponding authors, e-mail: andrea.giometto@gmail.com (ORCID ID: 0000-00020544-6023) and florian.altermatt@eawag.ch (ORCID ID: 0000-0002-4831-6958). 


\section{Appendix 1: Additional Methods and Results}

\section{Additional Methods}

\subsection{Autocorrelation length of the telegraph process}

723 Here we illustrate the connection between the autocorrelation length $c_{L}$ of the telegraph process (see section Methods, Model), its rate $\mu$ and the mean length of favorable and unfavorable patches $1 / \mu$. Let $I(x) \in\{0,1\}$ be the value of the telegraph process at the spatial coordinate $x$. $I(x)$ is composed of consecutive patches of $0 \mathrm{~s}$ and $1 \mathrm{~s}$ with lengths drawn randomly from an exponential distribution with rate $\mu$. Therefore, the mean patch length is $1 / \mu$. The number of times that $\mathrm{I}(\mathrm{x})$ switches between the two possible values $\{0,1\}$ in $\left(\mathrm{x}_{1}, \mathrm{x}_{2}\right)$ is a Poisson number with rate $\mu\left(\mathrm{x}_{2}-\mathrm{x}_{1}\right)$.

Therefore, $\mathrm{I}\left(\mathrm{x}_{1}\right)=\mathrm{I}\left(\mathrm{x}_{2}\right)$ with probability $\frac{1}{2}\left[1+\mathrm{e}^{-2 \mu\left(\mathrm{x}_{2}-\mathrm{x}_{1}\right)}\right]$, which is the probability that the

Poisson random number is even. Because $\mathrm{P}[\{\mathrm{I}(\mathrm{x})=1\}]=\mathrm{P}[\{\mathrm{I}(\mathrm{x})=0]=1 / 2$, the autocorrelation function of $\mathrm{I}(\mathrm{x})$ is equal to

$\mathrm{E}\left[\mathrm{I}\left(\mathrm{x}_{1}\right) \mathrm{I}\left(\mathrm{x}_{2}\right)\right]-\mathrm{E}[\mathrm{I}]^{2}=(+1) \cdot \mathrm{P}\left[\left\{\mathrm{I}\left(\mathrm{x}_{1}\right)=1\right\}\right] \cdot \mathrm{P}\left[\left\{\mathrm{I}\left(\mathrm{x}_{2}\right)=\mathrm{I}\left(\mathrm{x}_{1}\right)\right\}\right]-\frac{1}{4}=\frac{1}{4} \mathrm{e}^{-2 \mu\left(\mathrm{x}_{2}-\mathrm{x}_{1}\right)}$,

733

734

735

736

737

738

739

740

741

742

743

744

745

which is an exponentially decaying function with autocorrelation length $c_{L}=1 /(2 \mu)$.

\subsection{Numerical integration}

Equation (2) must be discretized in space to be numerically integrated. A suitable spatial discretization reads (Dornic et al. 2005, Giometto et al. 2014):

$\frac{\mathrm{d} \rho_{\mathrm{i}}}{\mathrm{dt}}(\mathrm{t})=\frac{1}{(\Delta \mathrm{x})^{2}}\left[\rho_{\mathrm{i}+1}(\mathrm{t})+\rho_{\mathrm{i}-1}(\mathrm{t})-2 \rho_{\mathrm{i}}(\mathrm{t})\right]+\mathrm{r}_{\mathrm{i}} \rho_{\mathrm{i}}(\mathrm{t})\left[1-\rho_{\mathrm{i}}(\mathrm{t})\right]+\frac{\sigma}{\sqrt{\Delta \mathrm{x}}} \sqrt{\rho_{\mathrm{i}}(\mathrm{t})} \eta_{\mathrm{i}}(\mathrm{t})$

where $\mathrm{i}$ identifies the lattice site, the term $\sqrt{\Delta \mathrm{x}}$ ensures proper normalization in the continuum limit (Doering et al. 2005) and $r_{i}=\delta_{I_{i}, 1}$ depends on the local value of the resource profile I (here, $\delta$ is the Kronecker's delta). The split-step method proposed in Dornic et al. (2005), which ensures that the density is never negative, was used to solve equation (A2). The spatial step in the numerical integration of equation (A2) was set to $\Delta \mathrm{x}=0.5$, while the temporal step was chosen equal to $\Delta \mathrm{t}=0.1$. The Courant-Friedrichs-Lewy condition for the diffusion equation $\Delta \mathrm{t} / \Delta \mathrm{x}^{2}<1$ was thus satisfied and $\Delta \mathrm{t} / \Delta \mathrm{x}<1$. The numerical integration of equation (A2) with $\sigma=0$ was 
performed using the same numerical scheme, modified in the choice of $\rho^{*}$ (we refer the reader to Dornic et al. 2005 for notation and symbols), which in the deterministic case is $\rho^{*}=\frac{\alpha}{\beta\left(\mathrm{e}^{\beta \Delta t}-1\right)}+$ $\rho \mathrm{e}^{\beta \Delta \mathrm{t}}$. The deterministic equation was integrated with three choices of the growth rate $\mathrm{r}$ in unfavorable regions of the landscape (where $\mathrm{I}=0$ ), specifically $\mathrm{r}=0, \mathrm{r}=-0.01$ and $\mathrm{r}=-0.1$. None of these choices for $r$ produced a slowdown of the front at large resource autocorrelation lengths, compared to small ones.

\section{The spatial discretization of equation (Fehler! Verweisquelle konnte nicht gefunden} werden.) reads:

$$
\begin{aligned}
& \frac{d \rho_{i}}{d t}(t)=\frac{D}{(\Delta x)^{2}}\left[\rho_{i+1}(t)+\rho_{i-1}(t)-2 \rho_{i}(t)\right]+r_{i} \rho_{i}(t)\left[1-\rho_{i}(t)\right]-\frac{1}{2 \Delta x}\left[g_{i+1} \rho_{i+1}(t)-\right. \\
& \left.g_{i-1} \rho_{i-1}(t)\right]+r_{i} \rho_{-} i\left[1-\frac{\rho_{i}(t)}{K}\right]+\frac{\sigma}{\sqrt{\Delta x}} \sqrt{\rho_{i}(t)} \eta_{i}(t), \quad(A 3)
\end{aligned}
$$

where $\mathrm{g}=\mathrm{d} \varphi / \mathrm{dx}[\mathrm{I}(\mathrm{x})]$. The split-step method proposed in Dornic et al. (2005) was modified to solve equation (A3), which contains an advection term that might cause an artificial loss of mass if the step sizes are too coarse. Such issue does not occur with the step sizes $\Delta x=0.6 \mathrm{~cm}$ and $\Delta \mathrm{t}=0.5 \mathrm{~min}^{-1}$ chosen here. The Courant-Friedrichs-Lewy condition for the diffusion equation was satisfied and $\Delta \mathrm{t} / \Delta \mathrm{x}<1$. Various alternatives to the spatial discretization (A3) were investigated, including higher-order discretizations of the spatial derivatives and upwind/downwind schemes for the advection term. Their performances were compared by imposing $r=0$ and small $\sigma$ and by inspecting mass conservation in a spatial window of length $12 \mathrm{~cm}$, with a light intensity profile corresponding to that generated by one LED located at the center of the spatial window. The discretization (A3) proved to be most effective in conserving the total mass $\left(\sum_{\mathrm{i}} \rho_{\mathrm{i}}\right)$ in the system and was therefore employed here.

\subsection{Square-root multiplicative noise}

The square root noise term in equations (Fehler! Verweisquelle konnte nicht gefunden werden.) and (Fehler! Verweisquelle konnte nicht gefunden werden.) is used to model demographic stochasticity (see, e.g., Dornic et al. (2005), Bonachela et al. (2012), Giometto et al. (2014), Villa Martín et al. 2015). The origin of the square root noise term in the equation can be 
understood in a simplified example where one considers the (local) process of birth $\mathrm{A} \rightarrow 2 \mathrm{~A}$ (where A represents an individual and the birth process represents cell duplication, in analogy with the experiment) and the (local) death process $A \rightarrow \varnothing$. The chemical Langevin equation for such two processes (Gillespie 2000), assuming birth and death rates $a_{1}(\rho)=b \rho$ and $a_{2}(\rho)=d \rho$ $\operatorname{reads} \frac{\mathrm{d} \rho}{\mathrm{dt}(\mathrm{t})}=\mathrm{a}_{1}[\rho(\mathrm{t})]-\mathrm{a}_{2}[\rho(\mathrm{t})]+\sqrt{\mathrm{a}_{1}(\rho)} \Gamma_{1}(\mathrm{t})-\sqrt{\mathrm{a}_{2}(\rho)} \Gamma_{2}(\mathrm{t})=\operatorname{r} \rho(\mathrm{t})=\sigma \sqrt{\rho} \Gamma(\mathrm{t})$, where $\rho$ denotes the density of individuals, $r=b-d \sigma=\sqrt{b+d}$ and $\Gamma_{1}(t), \Gamma_{2}(t)$ and $\Gamma(t)$ are $\delta$ correlated Gaussian white noises. As usual in the case of particle systems, the amplitude of the noise term is proportional to the square root of the density and thus noise dominates over any other term when the density is close to zero.

\section{Additional Results}

\subsection{Mean front propagation speed in heterogeneous landscapes}

Here we derive an approximation to the front propagation speed in the model equation (1), valid for large autocorrelation lengths and $\sigma$. We divide equation (1) by $K$ and $r_{0}$ and rescale time as $\mathrm{t}^{\prime}=\mathrm{r}_{0} \mathrm{t}$, which gives:

$$
\frac{\partial \rho^{\prime}\left(\mathrm{x}, \mathrm{t}^{\prime}\right)}{\partial \mathrm{t} \prime}=\frac{\mathrm{D}}{\mathrm{r}_{0}} \frac{\partial^{2} \rho^{\prime}\left(\mathrm{x}, \mathrm{t}^{\prime}\right)}{\partial \mathrm{x}^{2}}+\chi_{\mathrm{I}}(\mathrm{x}) \rho^{\prime}\left(\mathrm{x}, \mathrm{t}^{\prime}\right)\left[1-\rho^{\prime}\left(\mathrm{x}, \mathrm{t}^{\prime}\right)\right]+\frac{\sigma^{\prime}}{\sqrt{\mathrm{r}_{0}}} \sqrt{\rho^{\prime}\left(\mathrm{x}, \mathrm{t}^{\prime}\right)} \eta\left(\mathrm{x}, \mathrm{t}^{\prime}\right),
$$

where $\rho^{\prime}=\rho / K, \sigma^{\prime}=\sigma / \sqrt{\mathrm{K}}$ and $\chi_{\mathrm{I}}$ is the indicator function of the set of $\mathrm{x}$ for which $\mathrm{I}(\mathrm{x})>0$. We can further rescale space as $\mathrm{x}^{\prime}=\sqrt{\mathrm{D} / \mathrm{r}_{0}}$ and rewrite equation (1) as:

$\frac{\partial \rho^{\prime}\left(\mathrm{x}^{\prime}, \mathrm{t}^{\prime}\right)}{\partial \mathrm{t} \prime}=\frac{\partial^{2} \rho \prime\left(\mathrm{x}^{\prime}, \mathrm{t}^{\prime}\right)}{\partial \mathrm{x}^{\prime}}+\chi_{\mathrm{I}}\left(\mathrm{x}^{\prime}\right) \rho^{\prime}\left(\mathrm{x}^{\prime}, \mathrm{t}^{\prime}\right)\left[1-\rho^{\prime}\left(\mathrm{x}^{\prime}, \mathrm{t}^{\prime}\right)\right]+\sigma^{\prime \prime} \sqrt{\rho^{\prime}\left(\mathrm{x}^{\prime}, \mathrm{t}^{\prime}\right)} \eta\left(\mathrm{x}^{\prime}, \mathrm{t}^{\prime}\right)$,

where $\sigma^{\prime \prime}=\sigma^{\prime} /(\mathrm{rD})^{1 / 4}$. In the following we will study the front propagation speed in the rescaled equation (Fehler! Verweisquelle konnte nicht gefunden werden.), where we drop primes for convenience, one can recover the original dimensions by multiplying $\mathrm{t}$ by $\mathrm{r}_{0}$ and $\mathrm{x}$ by $\sqrt{\mathrm{r}_{0} / \mathrm{D}}$. The rationale for our approximation of the mean front propagation speed is as follows. Let $\mathrm{L}$ be the finite length of a landscape and $\mathrm{T}$ the time taken by the population to reach the end of such landscape $(x=L)$, starting from a localized initial condition at $x=0$. For large values of 
autocorrelation length $\mathrm{c}_{\mathrm{L}}$ and large enough $\sigma$, due to the local extinctions caused by demographic stochasticity, most of the time $\mathrm{T}$ is spent by the population trying to cross long patches of the landscape where $r=I=0$. We can therefore approximate the mean front propagation speed for large $c_{L}$ by computing the mean time that the front takes to cross an unfavorable patch of finite length z. Of course, such approximation is only valid when the waiting times dominate over the typical time scale of front propagation in favorable regions of the landscape. Therefore, the approximation can only hold for large enough values of the strength of demographic stochasticity $\sigma$.

\subsubsection{Propagation past a patch of unfavorable landscape}

We computed numerically the mean time $\langle\tau\rangle$ taken by the front to cross a region of landscape where $\mathrm{I}=0$, for different spatial extents of such region and different values of $\sigma$. We integrated numerically equation (2) in landscapes with resource profile $I(x)=\Theta(x-z)$, where $\Theta$ is the Heaviside step function. Such landscapes consist of a resource profile $I(x)=r(x)=1$, except for $\mathrm{x} \in[0,1]$, that is a finite patch of spatial extent $\mathrm{z}$ at the left end of the landscape, where $\mathrm{I}(\mathrm{x})=0$. The initial condition was $\rho(x, 0)=0$ for $x>0$ and $\rho(0,0)=k$, where $k$ is the mean population density computed numerically by integrating equation (2) in a landscape of spatial extent $\mathrm{L}=100$ with growth rate profile $r(x)=1$ for all $x \in[0, L]$. We fixed the Dirichlet boundary condition $\rho(0, t)=k$ and reflecting boundary conditions in $x=L$. We computed the mean time taken by the front to cross such unfavorable patch by measuring the first occurrence of $\rho(z)>10^{-3} \mathrm{k}$ in time. Fig. 1A shows the mean time $\langle\tau\rangle$ taken by the population to cross unfavorable patches of various extents z, computed for various values of $\sigma$. Such mean time $\langle\tau\rangle$ is a monotonically increasing function of both $\mathrm{z}$ and $\sigma$. To characterize the functional dependence of $\langle\tau\rangle(\mathrm{z}, \sigma)$ on $\mathrm{z}$ and $\sigma$, we note that in the limit $\sigma=0$ the dependence of $\langle\tau\rangle$ on $\mathrm{z}$ is that of the deterministic diffusion equation with boundary condition $\rho(0, t)=1$, that is, $\tau(z, 0)=\mathrm{Cz}^{2}$, where $\mathrm{C}$ is the solution of $\operatorname{erfc}(1 /$ $[2 \sqrt{\mathrm{C}}])=10^{-3}$, where erfc is the complementary error function. We assume that $\langle\tau\rangle(\mathrm{z}, \sigma)$ depends on $\mathrm{z}$ and $\sigma$ through the functional form:

$\langle\tau\rangle(\mathrm{z}, \sigma)=C \mathrm{z}^{2} \mathbf{F}\left(\mathrm{z} \sigma^{\mathrm{b}}\right)$ 


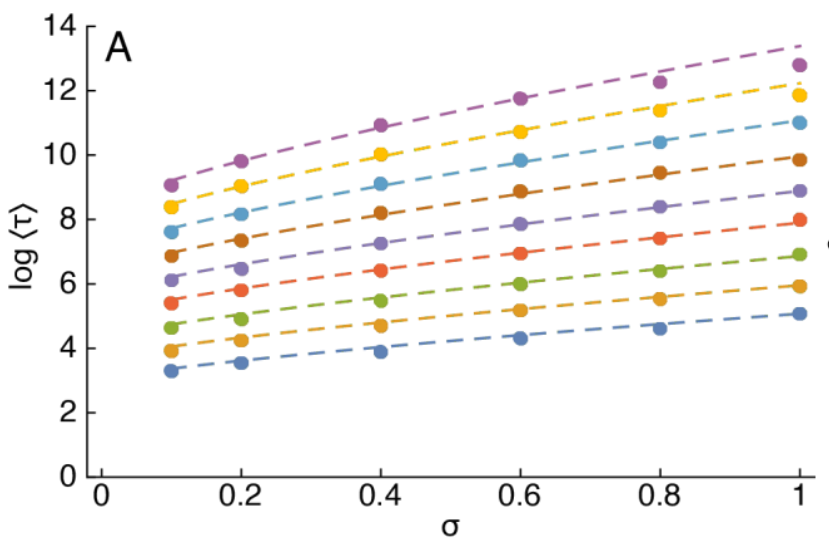

$\langle\tau\rangle(z, \sigma)=C z^{2} e^{d\left(z \sigma^{b}\right)^{a}}$

where $\mathbf{F}(\mathrm{x})$ is a function that goes to the constant 1 for $\mathrm{x} \rightarrow 0$. We can verify the validity of equation (Fehler! Verweisquelle konnte nicht gefunden werden.) by plotting $\mathrm{z}^{-2} \tau$ versus $z \sigma^{\mathrm{b}}$ and varying $b$. Because we are able to find a value of $b=b^{*}$ for which data from the numerical integrations collapse onto one single curve (Fig. 1B), the assumption on the functional form of $\langle\tau\rangle$ is verified. To further identify the functional dependence of $\langle\tau\rangle$ on $\mathrm{z}$ we plotted $\log \left[\log \left(\mathrm{z}^{-2}\langle\tau\rangle\right)-\right.$ $\log \mathrm{C}] \mathrm{vs} \log \left(\mathrm{z} \sigma^{\mathrm{b}}\right)$ and observed that simulation data aligned along a straight line. Therefore, our numerical analysis suggests that the functional dependence of $\tau$ on $\mathrm{z}$ and $\sigma$ is given by:

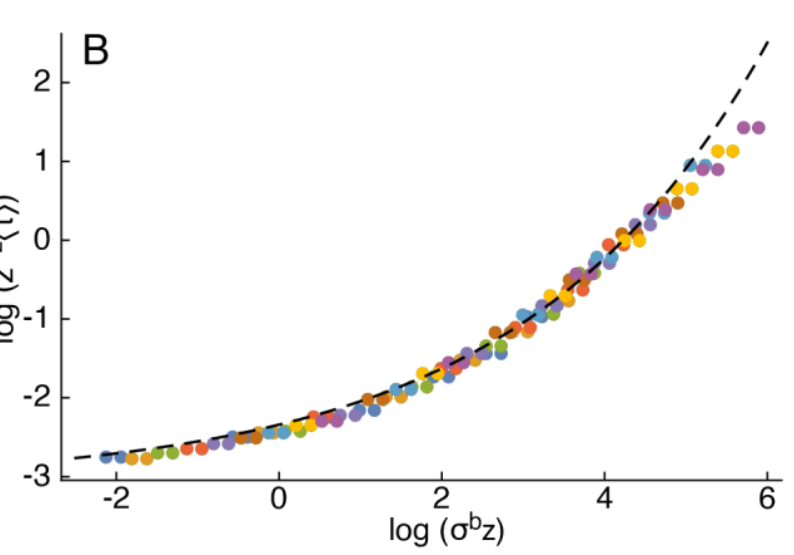

836 Figure 1: Mean time $\langle\tau\rangle$ taken by a diffusing population subject to demographic stochasticity to cross patches of length $\mathrm{z}$, calculated for different values of $\mathrm{z}$ and $\sigma$ across 192 integrations of equation (2). (A) $\tau$ is a monotonically increasing function of $\mathrm{z}$ and $\sigma$. Dots of identical color were computed with identical $\mathrm{z}=21,29,49,57,79,111,156$ and 218, from bottom to top. Lines are computed via equation (Fehler! Verweisquelle konnte nicht gefunden werden.), the color code identifies the value of $\mathrm{z}$ as for the dots. (B) Simulation data collapse onto the same curve when $\mathrm{z}^{-2} \tau$ is plotted against $\sigma^{\mathrm{b}} \mathrm{z}$, proving the assumption made in equation (Fehler! Verweisquelle konnte nicht gefunden werden.). Dots are color-coded as in panel (A), the dashed black line

844 shows the function $\mathbf{F}$ computed according to equation (Fehler! Verweisquelle konnte nicht 845

\section{gefunden werden.).}


We estimated $b$ by maximizing the $\mathrm{R}^{2}$ (coefficient of determination) of the least-squares linear

847 fit of $\log \left[\log \left(\mathrm{z}^{-2}\langle\tau\rangle\right)-\log C\right]$ versus $\log \left(z \sigma^{\mathrm{b}}\right)$. The slope and intercept of the linear fit with

848 maximum $\mathrm{R}^{2}$ gave the estimate of a and d. Fig. 1 shows that equation (Fehler! Verweisquelle

849 konnte nicht gefunden werden.) reproduces the numerical data satisfactorily with the

850 parameters $\mathrm{d}=0.74, \mathrm{a}=0.34$ and $\mathrm{b}=2.25$, identified as outlined above.

\subsubsection{Approximation for the mean front propagation speed in heterogeneous landscapes}

For large values of the autocorrelation length $c_{L}=1 /(2 \mu)(\mu$ is the rate of the telegraph process used to generate the heterogeneous landscapes, see Methods), most of the time taken by the front to propagate through a landscape of length $\mathrm{L}$ is spent trying to cross finite stretches of the landscape where $\mathrm{r}=\mathrm{I}=0$. We can therefore approximate the front propagation speed as $\mathrm{v}=\mathrm{L} / \mathrm{T}=\mathrm{L} / \sum_{\mathrm{i}=1}^{\mathrm{N}}\langle\tau\rangle\left(\mathrm{z}_{\mathrm{i}}, \sigma\right)$ (black dots in Fig. Fehler! Verweisquelle konnte nicht gefunden werden.), where $\mathrm{N}$ is the number of unfavorable patches in $\mathrm{x} \in[0, \mathrm{~L}]$ (of extent $\mathrm{z}_{\mathrm{i}}$ ) and $\langle\tau\rangle$ is the mean time taken to cross a patch of spatial extent $\mathrm{z}_{\mathrm{i}}$, estimated via equation (Fehler! Verweisquelle konnte nicht gefunden werden.). In landscapes where unfavorable patches of length $\mathrm{z}$ occur with probability $\mu \mathrm{e}^{-\mu \mathrm{z}} \mathrm{dz}$, one can approximate the mean front propagation speed for large autocorrelation length $\mathrm{c}_{\mathrm{L}}$ as:

$$
\mathrm{V}=\frac{\mathrm{L}}{\frac{\mu \mathrm{L}}{2} \int_{0}^{\mathrm{L}} \mathrm{dz}\langle\tau\rangle(\mathrm{z}, \sigma) \mu \mathrm{e}^{-\mu \mathrm{z}}} \simeq \frac{8 \mathrm{c}_{\mathrm{L}}^{2}}{\int_{0}^{\infty} \mathrm{dz}\langle\tau\rangle(\mathrm{z}, \sigma) \mathrm{e}^{-\frac{\mathrm{z}}{2 \mathrm{c}_{\mathrm{L}}}}}
$$
where $\langle\tau\rangle(\mathrm{z}, \sigma)$ is given by equation (Fehler! Verweisquelle konnte nicht gefunden werden.) and $\mu \mathrm{L} / 2$ at the denominator is the average number of unfavorable patches in the landscape. If $\mathrm{L}$ is comparable to $c_{\mathrm{L}}$, one can substitute $\mu \mathrm{L} / 2$ with a more precise estimate, which is given in the next section. Fig. Fehler! Verweisquelle konnte nicht gefunden werden. shows that equation (Fehler! Verweisquelle konnte nicht gefunden werden.) gives a good approximation to the front propagation velocities computed in the numerical integrations, for large values of $c_{L}$. 


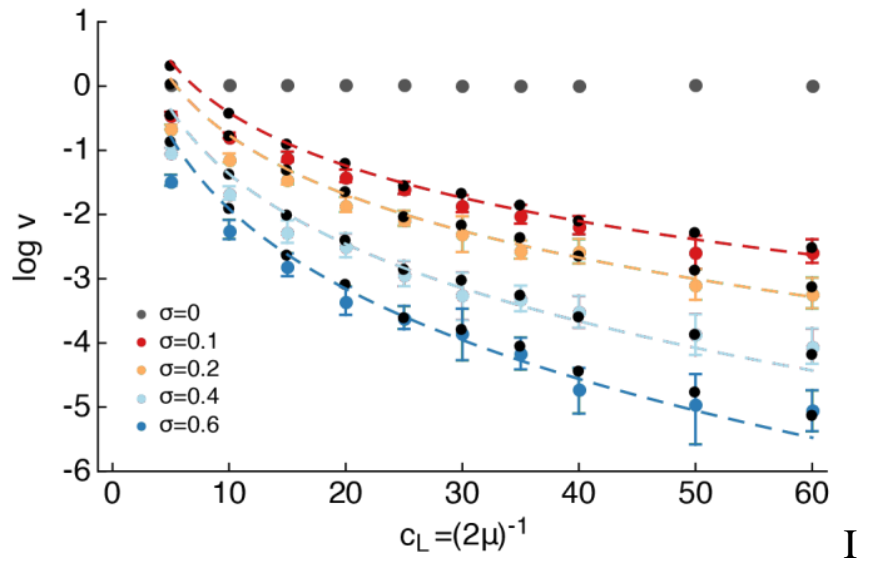

871 Figure 2: The mean front speed $\mathrm{v}$ decreases with increasing resource autocorrelation length

$872 \mathrm{c}_{\mathrm{L}}=1 /(2 \mu)$ ( $\mu$ is the rate of the telegraph process used to generate the heterogeneous landscapes)

873 and can be approximated by equation (Fehler! Verweisquelle konnte nicht gefunden werden.)

874 for large $\mathrm{c}_{\mathrm{L}}$ (dashed lines). Colored data points highlight the mean speed $\mathrm{v}$ computed by

875 numerically integrating equation (2) and by fitting the mean front position versus time to a

876 straight line. Different colors refer to different values of $\sigma$ according to the legend. Error bars

877 display the $95 \%$ confidence interval for $\mathrm{v}$, computed with $2 \cdot 10^{3}$ bootstrap samples. Error bars for

$878 \sigma=0$ are smaller than symbols. Dashed lines are the mean front speed computed according to

879 equation (Fehler! Verweisquelle konnte nicht gefunden werden.). Black dots are the

880 approximation to the mean front speed computed as $v=L / T=L / \sum_{i \in Z}\langle\tau\rangle\left(z_{i}, \sigma\right)$, where $Z$ is the

881 set of unfavorable windows in the numerical landscapes. Dashed lines and black dots may differ

882 because the numerical landscapes were finite, thus the distribution of unfavorable window lengths

883 may differ slightly from the exponential pdf with typical length $1 / \mu=c_{L}$. 


\subsubsection{Correction to the average number of patches if $L$ is comparable to $c_{L}$.}

886

887

888

889

890

891

892

893

894

895

896

897

898

899

900

901

902

903

904

905

906

907

908

We provide here a correction to the term $\mu \mathrm{L} / 2$ at the denominator of equation (Fehler!

Verweisquelle konnte nicht gefunden werden.), which is relevant when $L \simeq C_{L}$. If the first patch at $x=0$ is favorable (i.e., $r>0$ ), the average number of unfavorable patches in a landscape of length $\mathrm{L}$ can be computed as follows. Let $\mathrm{z}_{\mathrm{i}}$ be the rightmost coordinate of each patch in the landscape. The average number of unfavorable patches is equal to:

$\langle N\rangle=\sum_{n=1}^{\infty} n P\left[z_{2 n}<L \cap z_{2 n+1} \geq L\right]+\sum_{n=1}^{\infty} n P\left[z_{2 n-1}<L \cap z_{2 n} \geq L\right]$.

Using properties of the exponential distribution of patch lengths one has:

$\mathrm{P}\left[\mathrm{z}_{2 \mathrm{n}}<\mathrm{L} \cap \mathrm{z}_{2 \mathrm{n}+1} \geq \mathrm{L}\right]=$

$\mu^{2 n+1} \int_{0}^{L} d z_{1} e^{-\mu z_{1}} \int_{z_{1}}^{L} d z_{2} e^{-\mu\left(z_{2}-z_{1}\right)} \cdots \int_{z_{2 n-1}}^{L} d z_{2 n} e^{-\mu\left(z_{2 n}-z_{2 n-1}\right)} \int_{L}^{\infty} d z_{2 n+1} e^{-\mu\left(z_{2 n+1}-z_{2 n}\right)}=$

$\frac{\mathrm{e}^{-\mu \mathrm{L}}}{(2 \mathrm{n}) !}(\mu \mathrm{L})^{2 \mathrm{n}}$

$\mathrm{P}\left[\mathrm{z}_{2 \mathrm{n}-1}<\mathrm{L} \cap \mathrm{z}_{2 \mathrm{n}} \geq \mathrm{L}\right]=$

$\mu^{2 n} \int_{0}^{L} d z_{1} e^{-\mu z_{1}} \int_{z_{1}}^{L} d z_{2} e^{-\mu\left(z_{2}-z_{1}\right)} \cdots \int_{z_{2 n-2}}^{L} d z_{2 n-1} e^{-\mu\left(z_{2 n-1}-z_{2 n-2}\right)} \int_{L}^{\infty} d z_{2 n+1} e^{-\mu\left(z_{2 n}-z_{2 n-1}\right)}=$ $\frac{\mathrm{e}^{-\mu \mathrm{L}}}{(2 \mathrm{n}-1) !}(\mu \mathrm{L})^{2 \mathrm{n}-1}$,

and therefore:

$\langle N\rangle=\sum_{n=1}^{\infty} n\left[\frac{e^{-\mu L}}{(2 n) !}(\mu L)^{2 n}+\frac{e^{-\mu L}}{(2 n-1) !}(\mu L)^{2 n-1}\right]=\frac{\mu L}{2}+\frac{e^{-\mu L}}{2} \sinh (\mu L)$,

where sinh is the hyperbolic sine function. One can repeat the same analysis in the case where the first patch at $\mathrm{x}=0$ is unfavorable (i.e., $r=0$ ). In this case one finds:

$\langle N\rangle=\sum_{n=1}^{\infty} n\left[\frac{e^{-\mu L}}{(2 n-2) !}(\mu L)^{2 n-2}+\frac{e^{-\mu L}}{(2 n-1) !}(\mu L)^{2 n-1}\right]=\frac{\mu L}{2}+\frac{3}{4}+e^{-\mu L}$,

Finally, if the first patch is favorable or unfavorable with equal probabilities, then:

$\langle\mathrm{N}\rangle=\frac{1}{2}\left[\frac{\mu \mathrm{L}}{2}+\frac{\mathrm{e}^{-\mu \mathrm{L}}}{2} \sinh (\mu \mathrm{L})\right]+\frac{1}{2}\left[\frac{\mu \mathrm{L}}{2}+\frac{3}{4}+\mathrm{e}^{-\mu \mathrm{L}}\right]$,

If $L \gg 2 / \mu=4 c_{L}$, the average number of unfavorable patches in a landscape of length $L$ tends to $\mu \mathrm{L} / 2$. 


\subsection{Fluctuations of the invasion time}

\section{2.2.1 Fluctuations of the time taken to cross a patch of unfavorable landscape}

911 In this section we study the fluctuations of the total invasion time in heterogeneous landscapes of

912 finite size L. To this end, we first characterize the standard deviation $\sigma_{\tau}$ of the time $\tau$ taken by a

913 diffusing population subject to demographic stochasticity to cross an unfavorable patch $(\mathrm{r}=0)$ of

914 spatial extent $z$. Inspection of the numerical results shows (Fig. 3B) that $\mathrm{z}^{-2} \sigma_{\tau}$ is a function of

$915 \mathrm{z}^{-2}\langle\tau\rangle(\mathrm{z}, \sigma)$, that is:

$916 \sigma_{\tau}(\mathrm{z}, \sigma)=\mathrm{z}^{2} \mathrm{~S}\left[\mathrm{z}^{-2}\langle\tau\rangle(\mathrm{z}, \sigma)\right]$

917

918
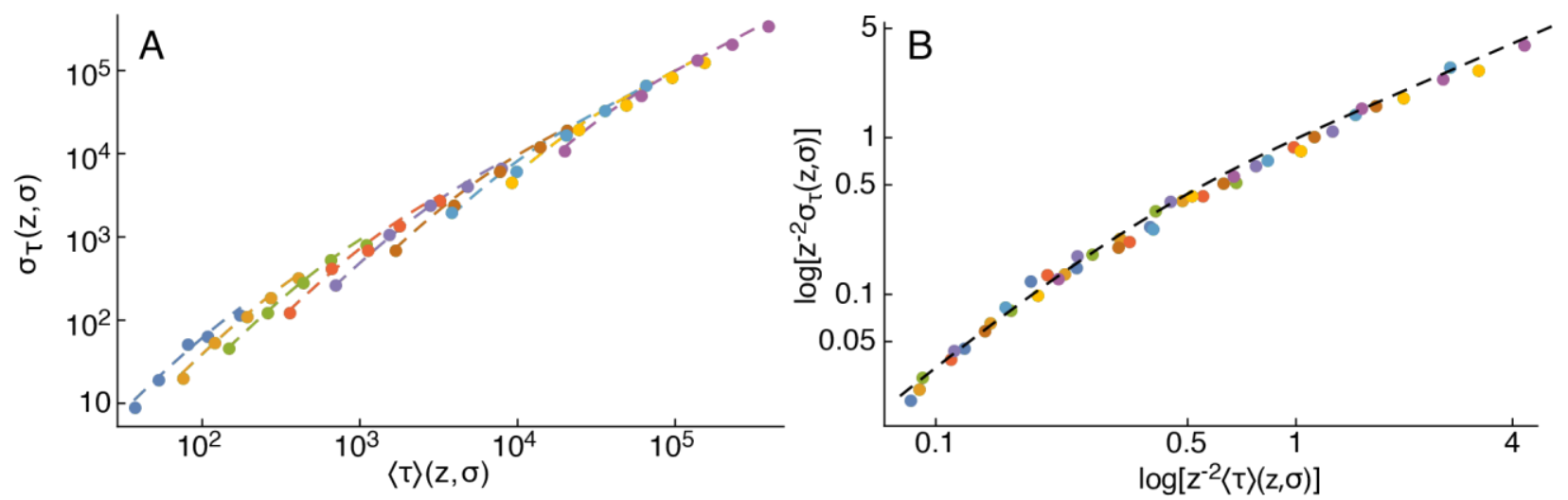

919 Figure 3: (A) Standard deviation $\sigma_{\tau}$ of the time taken by a diffusing population subject to

920 demographic stochasticity to cross patches of length $\mathrm{z}$, calculated for different values of $\mathrm{z}$ and $\sigma$

921 across 96 integrations of equation (Fehler! Verweisquelle konnte nicht gefunden werden.)

922 (double logarithmic plot). Different colors refer to different values of $\sigma$, from $\sigma=0.1$ (blue dots

923 at the bottom left corner) to $\sigma=1.4$ (violet dots at the top right corner). Dashed lines are

924 computed with equation (Fehler! Verweisquelle konnte nicht gefunden werden.). (B)

925 Simulation data collapse onto the same curve when $\mathrm{z}^{-2} \sigma_{\tau}$ is plotted against $\mathrm{z}^{-2}\langle\tau\rangle$, proving the

926 validity of equation (Fehler! Verweisquelle konnte nicht gefunden werden.). Dots are color-

927 coded as in panel (A), the dashed black line shows the function $\mathbf{S}$ computed according to equation

928 (Fehler! Verweisquelle konnte nicht gefunden werden.). 
where $\mathbf{S}(\mathrm{x})$ is a function that goes to 0 for $\mathrm{x} \rightarrow 0$. In fact, data from the numerical integrations of equation (2) in landscapes with resource profile $\mathrm{I}(\mathrm{x})=\Theta(\mathrm{x}-\mathrm{z})(\Theta$ is the Heaviside step function, the same numerical data were used to derive equation AFehler! Verweisquelle konnte nicht gefunden werden.) collapse on the same curve when $z^{-2} \sigma_{\tau}$ is plotted against $z^{-2}\langle\tau\rangle(z, \sigma)$ (Fig. 3B). The functional form:

$$
\sigma_{\tau}(\mathrm{z}, \sigma)=\langle\tau\rangle(\mathrm{z}, \sigma)\left[1-\mathrm{e}^{-\mathrm{kz}^{-2}\langle\tau\rangle(\mathrm{z}, \sigma)}\right],
$$

is found to provide a good fit to the numerical data, with the best-fit estimate of the coefficient $\mathrm{k}=4.17$ (dashed lines in Fig. 3).

\subsubsection{Fluctuations of the total invasion time in heterogeneous landscapes}

We can use equation (AFehler! Verweisquelle konnte nicht gefunden werden.) to approximate the variance of the total invasion time $T$ (i.e., the time after which the density $\rho(L, T)$ is larger than a threshold density value) in heterogeneous landscapes composed of favorable and unfavorable patches. In fact, the variance of the total invasion time in our simplified model, where we neglect the time spent by the front in propagating through favorable patches, and further assuming that the times spent to cross each unfavorable patch are independent from each other, is given by:

$\operatorname{Var}[\mathrm{T}]=\sum_{\mathrm{i}=1}^{\mathrm{N}} \sigma_{\tau}^{2}\left[\langle\tau\rangle\left(\mathrm{z}_{\mathrm{i}}, \sigma\right)\right]$

where $\mathrm{N}$ is the number of unfavorable patches in $\mathrm{x} \in[0, \mathrm{~L}]$ (patches of extent $\mathrm{z}_{\mathrm{i}}$ ) and $\sigma_{\tau}$ is given by equation (Fehler! Verweisquelle konnte nicht gefunden werden.). We show in Fig. 4 that equation (A11Fehler! Verweisquelle konnte nicht gefunden werden.) gives a good estimate of the variance of the total invasion time in heterogeneous landscapes. Details are provided in the figure caption. 
953

954

955

956

957

958

959

960

961

962

963

964

965

966

967

968

969

970
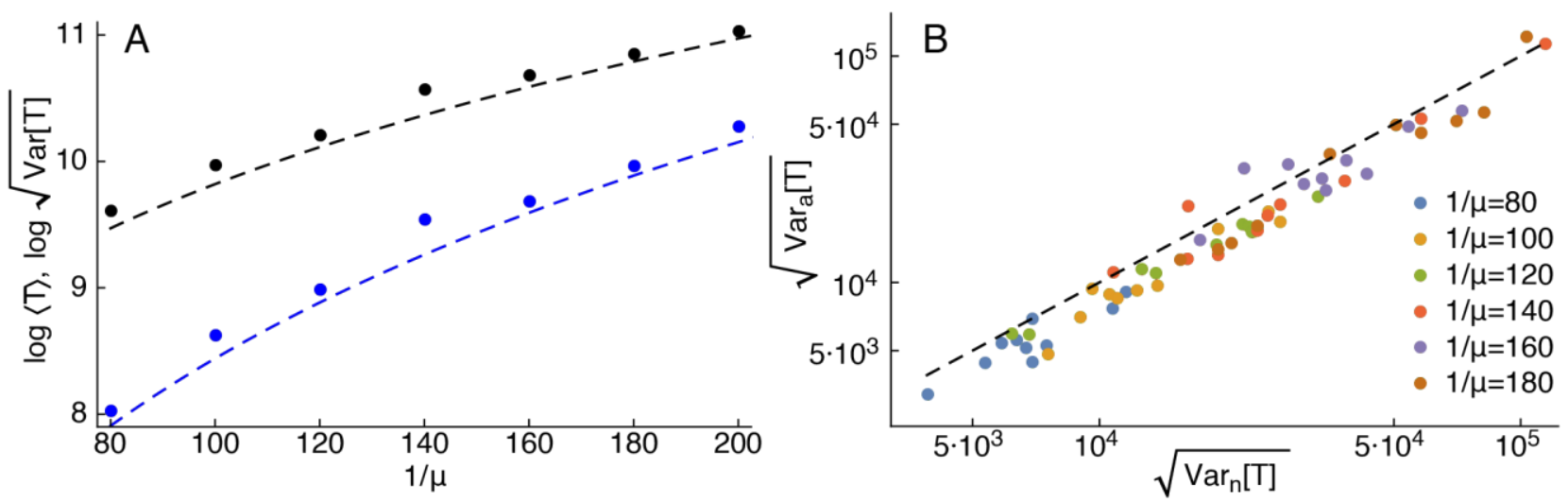

Figure 4: (A) Mean total time $\langle\mathrm{T}\rangle$ (black dots) of invasion and its standard deviation (blue dots) in numerical integrations of equation (2) in square-wave landscapes of length $\mathrm{L}=1400$, that is, landscapes composed of alternated favorable and unfavorable patches of length $1 / \mu$ (means and standard deviations were computed across 200 integrations for each value of $1 / \mu)$. The numerical estimates for $\langle\mathrm{T}\rangle$ and $\sqrt{\operatorname{Var}[\mathrm{T}]}$ are well approximated by the approximations $\langle\mathrm{T}\rangle=\frac{\mu \mathrm{L}}{2} \tau(1 / \mu, \sigma)$ (black dashed line) and by equation (A11Fehler! Verweisquelle konnte nicht gefunden werden.) (blue dashed line). (B) Numerically computed standard deviations $\sqrt{\operatorname{Var}_{\mathrm{n}}[\mathrm{T}]}$ (double logarithmic plot) of the total time $\mathrm{T}$ of invasion in numerical integrations of equation (2) in landscapes with exponentially distributed favorable and unfavorable patches are well approximated by the theoretical approximation $\sqrt{\operatorname{Var}_{\mathrm{a}}[\mathrm{T}]}$, computed according to equation (A11Fehler! Verweisquelle konnte nicht gefunden werden.). Each dot represents one landscape of length $\mathrm{L}=2000$ and mean patch length $1 / \mu$ according to the legend. Such landscapes were generated with the same procedure outlined in the Methods. To compute $\sqrt{\operatorname{Var}_{\mathrm{n}}[\mathrm{T}]}$, we performed 96 numerical integrations for each landscape. The dashed black line is the 1:1 line. Numerical estimates and theoretical approximations are calculated with $\sigma=0.4$ in both panels. 


\subsection{Front propagation at different mean resource densities}

972 Other works (e.g., Dewhirst and Lutscher 2009) have studied the propagation of invasion fronts in 973 landscapes with different average amounts of resources. One may wonder whether the slowdown

974 effect caused by varying autocorrelation lengths of the resource distribution might also be found 975 in landscapes endowed with mean percentages of suitable habitat different from $f_{1}=f_{0}=1 / 2$. To 976 show that such slowdown effect occurs also when the suitable and unsuitable habitats occur at 977 different frequencies throughout the landscape, we have integrated equation (2) on landscapes 978 endowed with various resource autocorrelation lengths and mean frequency of suitable (i.e., $r>0$ ) 979 and unsuitable (i.e., $r=0$ ) habitat equal to $f_{1}=1 / 2$ and $f_{0}=2 / 3$, respectively. Such landscapes were 980 generated as follows: we extracted the length of each favorable and unfavorable patch from 981 exponential distributions with rate $\mu_{1}=3 /\left(4 c_{L}\right)$ and $\mu_{0}=3 /\left(8 c_{L}\right)$, respectively, so that the resource 982 autocorrelation length was $\mathrm{c}_{\mathrm{L}}$ and the frequencies of favorable/unfavorable habitat were as 983 desired. Additionally, we have integrated equation (2) on the same landscapes switching each 984 favorable patch of the landscape with an unfavorable one, so that favorable habitats occurred with 985 frequency $f_{1}=2 / 3$ (and thus unfavorable habitats with frequency $f_{1}=1 / 3$ ). Fig. 5 shows that 986 increasing the mean frequency of suitable habitat increases the invasion speed, but the slowdown 987 effect caused by varying resource autocorrelation lengths is also present when favorable and 988 unfavorable habitats occur at frequencies different from 1/2. Furthermore, equation (5) can be used to approximate the mean speed of invasion for large $c_{\mathrm{L}}$ at values of $\mathrm{f}_{0}$ different from $1 / 2$, as

990 shown by the agreement between dashed lines and simulation data points in Fig. 5.

991 


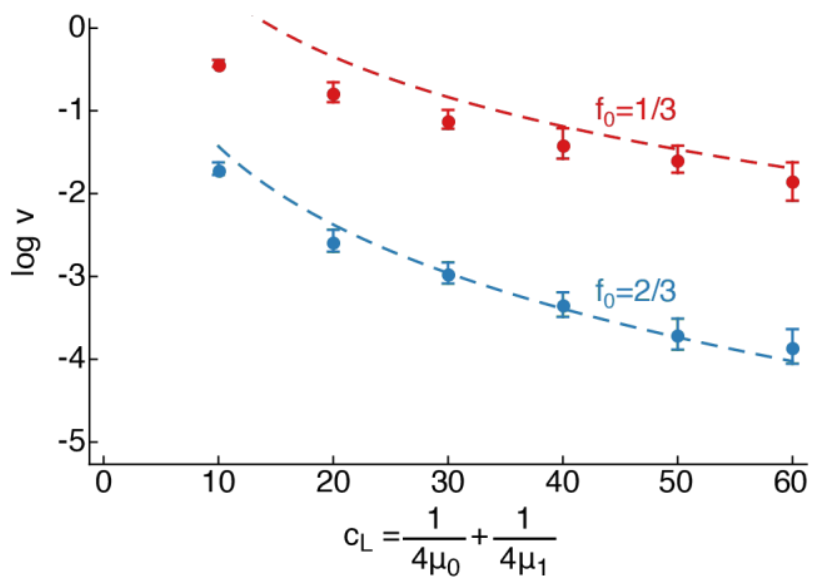

993 Figure 5: Mean front propagation speed in landscapes with favorable and unfavorable habitats 994 occurring at frequencies different from $\mathrm{f}_{1}=\mathrm{f}_{0}=1 / 2$. Red dots display the mean front speed in 96 995 replicated invasions in different landscapes with frequency of unsuitable habitat $\mathrm{f}_{0}=1 / 3$. favorable 996 patches lengths were distributed exponentially with rate $\mu_{1}=3 /\left(8 c_{L}\right)$ and unfavorable ones with 997 rate $\mu_{0}=3 /\left(4 c_{L}\right)$. Blue dots display the mean front speed in 96 replicated invasions in different 998 landscapes with frequency of unsuitable habitat $f_{0}=2 / 3$. Error bars display the $95 \%$ confidence 999 interval for $\mathrm{v}$, computed with $2 \cdot 10^{3}$ bootstrap samples. favorable patches lengths were distributed 1000 exponentially with rate $\mu_{1}=3 /\left(4 c_{L}\right)$ and unfavorable ones with rate $\mu_{0}=3 /\left(8 c_{L}\right)$. Dashed lines show 1001 mean front speeds approximated via equation (5) of the main text.

1002 


\section{Additional Tables}

1004 Table 1: Mixed-effect test statistics for all choices of density threshold $\bar{\rho}$.

\begin{tabular}{|c|c|c|c|c|c|c|}
\hline Threshold $\bar{\rho}$ & Value & & $\begin{array}{l}\text { Std. } \\
\text { Error }\end{array}$ & $\mathrm{df}$ & t-value & p-value \\
\hline $45 \mathrm{~cm}^{-1}$ & $\begin{array}{l}\text { Intercept } \\
\text { Autocorrelation length }\end{array}$ & $\begin{array}{l}57.15 \\
-11.31\end{array}$ & $\begin{array}{l}3.65 \\
4.94\end{array}$ & 94 & $\begin{array}{l}15.65 \\
-2.29\end{array}$ & $\begin{array}{l}p<10^{-4} \\
p=0.0480\end{array}$ \\
\hline $60 \mathrm{~cm}^{-1}$ & $\begin{array}{l}\text { Intercept } \\
\text { Autocorrelation length }\end{array}$ & $\begin{array}{l}45.98 \\
-11.61\end{array}$ & $\begin{array}{l}3.27 \\
4.43\end{array}$ & 94 & $\begin{array}{l}14.04 \\
-2.62\end{array}$ & $\begin{array}{l}p<10^{-4} \\
p=0.0279\end{array}$ \\
\hline $75 \mathrm{~cm}^{-1}$ & $\begin{array}{l}\text { Intercept } \\
\text { Autocorrelation length }\end{array}$ & $\begin{array}{l}45.27 \\
-9.65\end{array}$ & $\begin{array}{l}2.88 \\
3.90\end{array}$ & 94 & $\begin{array}{l}15.70 \\
-2.47\end{array}$ & $\begin{array}{l}p<10^{-4} \\
p=0.0355\end{array}$ \\
\hline $90 \mathrm{~cm}^{-1}$ & $\begin{array}{l}\text { Intercept } \\
\text { Autocorrelation length }\end{array}$ & $\begin{array}{l}36.65 \\
-9.04\end{array}$ & $\begin{array}{l}2.84 \\
3.85\end{array}$ & 94 & $\begin{array}{l}12.91 \\
-2.35\end{array}$ & $\begin{array}{l}p<10^{-4} \\
p=0.0433\end{array}$ \\
\hline $105 \mathrm{~cm}^{-1}$ & $\begin{array}{l}\text { Intercept } \\
\text { Autocorrelation length }\end{array}$ & $\begin{array}{l}35.91 \\
-10.79\end{array}$ & $\begin{array}{l}3.04 \\
4.11\end{array}$ & $\begin{array}{l}44 \\
9\end{array}$ & $\begin{array}{l}11.83 \\
-2.62\end{array}$ & $\begin{array}{l}p<10^{-4} \\
p=0.0276\end{array}$ \\
\hline
\end{tabular}

1005 Mixed-effect test statistics testing the speed of front propagation, with the autocorrelation length

1006 treatment as single fixed effect and time/replicate as random effect. The treatment with small

1007 autocorrelation length had 5 replicates, the treatment with large autocorrelation length had 6

1008 replicates. Different lines refer to different threshold values $\bar{\rho}$ at which the front position was

1009 measured.

1010 


\section{Additional Figures}

1012

1013

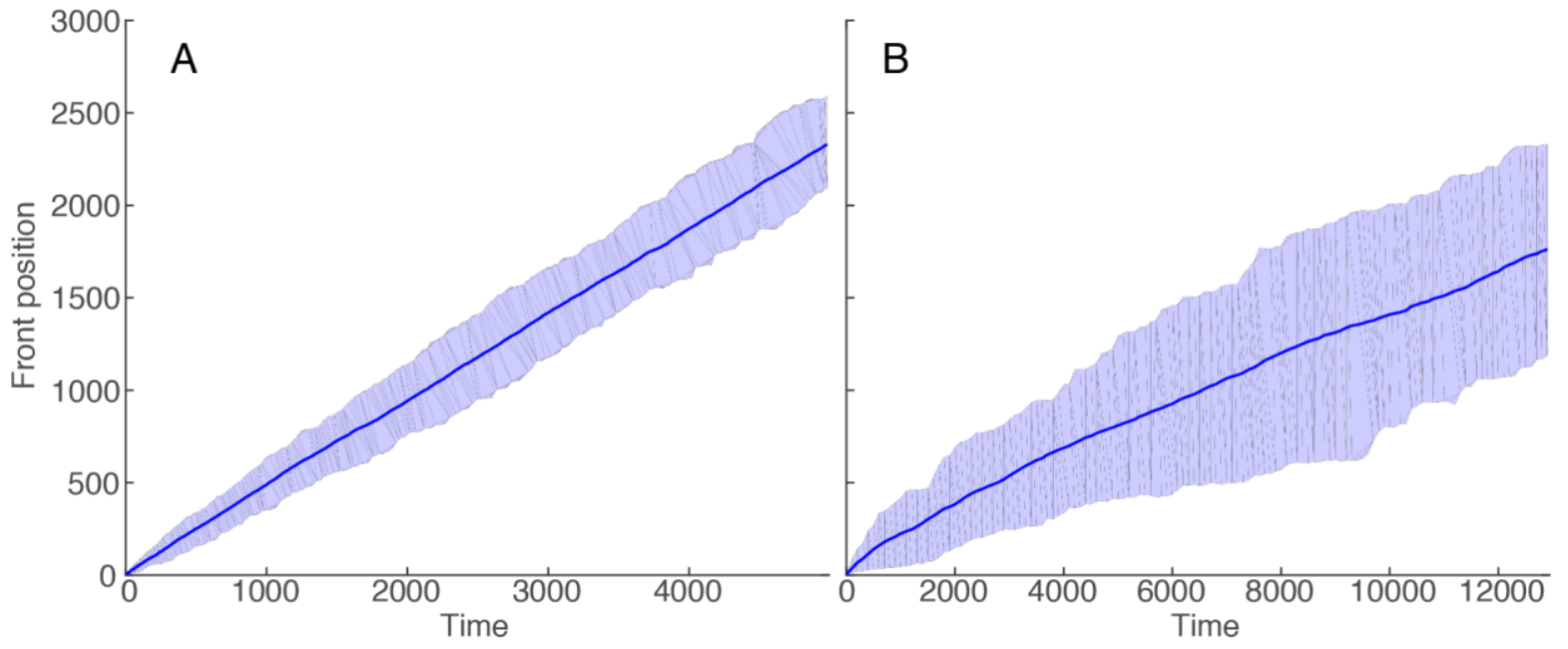

1014 Figure 6: Mean position of the front (blue lines) and 68\% confidence interval (shaded regions) in

1015 numerical integrations of the model equation (2) with $\sigma=0.1$ and resource autocorrelation lengths $1016 \quad c_{L}=5(A)$ and $c_{L}=20(B)$. 

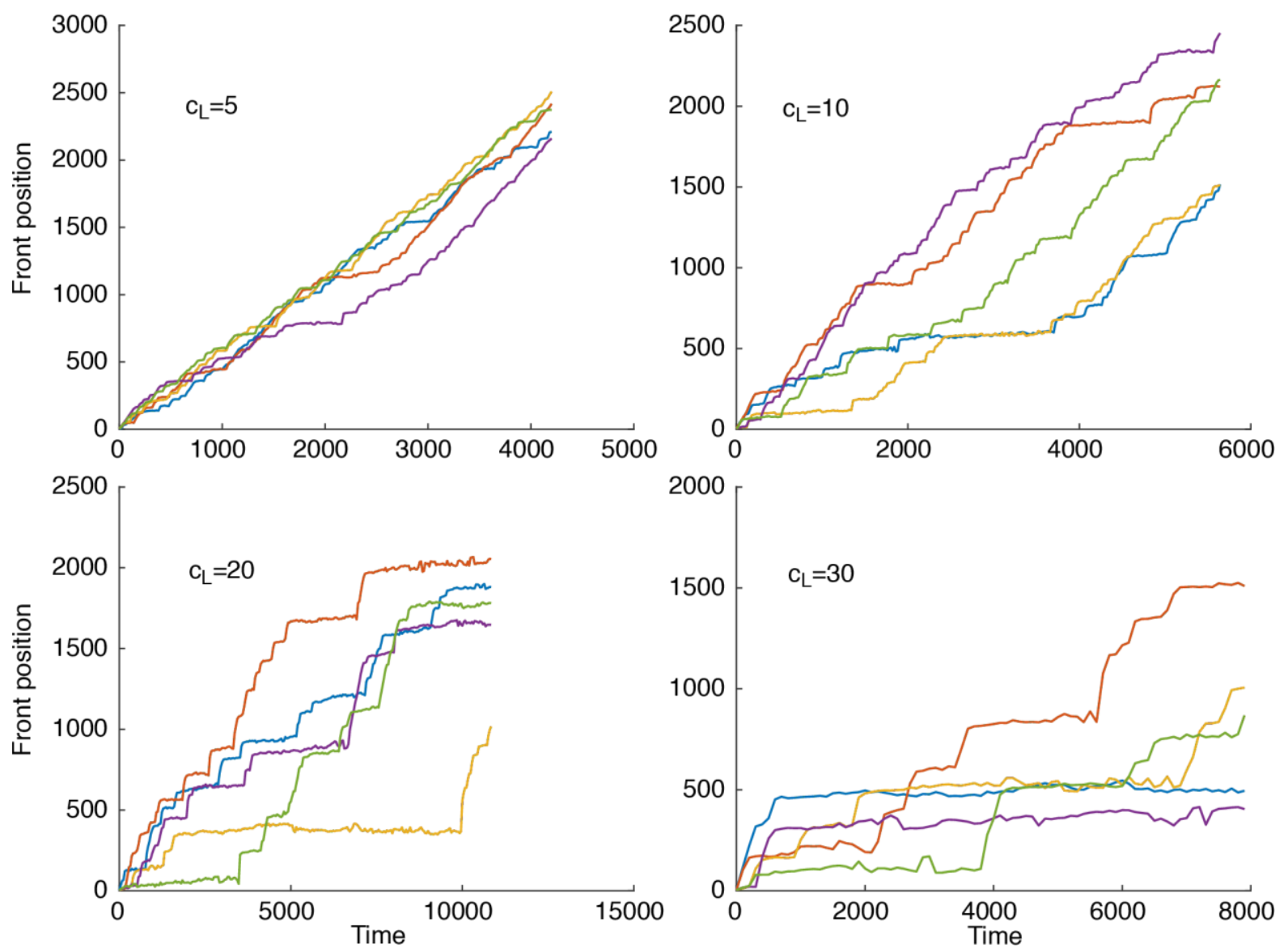

1018 Figure 7: Examples of front propagation in numerical integrations of the model (equation 2) in

1019 landscapes with different resource autocorrelation lengths $\mathrm{c}_{\mathrm{L}}$ and fixed amplitude of demographic 1020 stochasticity $\sigma=0.2$. 


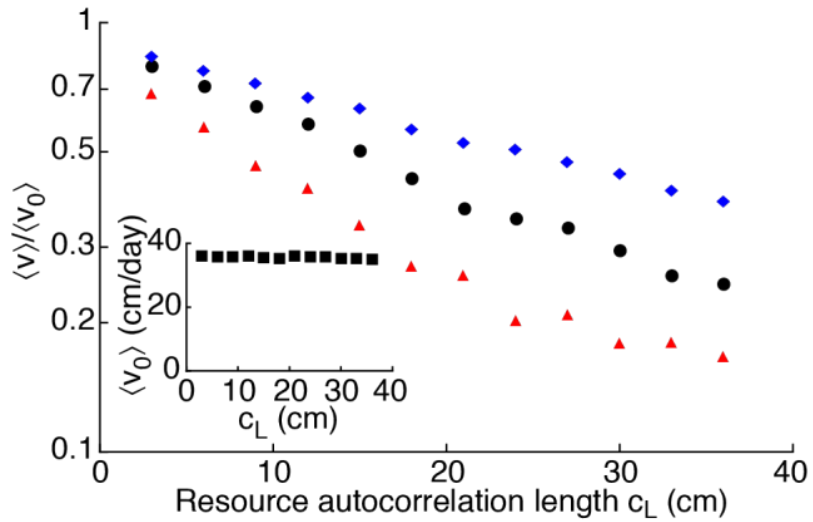

1023 Figure 8: Front propagation computed in numerical integrations of the model equation (Fehler!

1024 Verweisquelle konnte nicht gefunden werden.) (with spatial discretization equation A3). The

1025 mean invasion speed decreases with increasing resource autocorrelation length $\mathrm{c}_{\mathrm{L}}=\Delta \mathrm{L} /(2 \lambda)(\lambda$

1026 is the transition probability of the Markov Chain used to generate the heterogeneous landscapes

1027 and $\Delta \mathrm{L}$ is the experimental distance between LEDs, see Methods) and is a decreasing function of

1028 the amplitude of demographic stochasticity $\sigma$ (log-linear plot, black dots: $\sigma=0.4 \mathrm{~min}^{-1 / 2}$, red

1029 triangles: $\sigma=0.7 \mathrm{~min}^{-1 / 2}$ ). The mean speed of invasion is larger in the absence of directed

1030 movement towards resources (blue diamonds computed with $\sigma=0.4 \mathrm{~min}^{-1 / 2}$ and $\varphi=0$ ).

1031 Invasion speeds are reported here divided by the mean front speed $\left\langle\mathrm{v}_{0}\right\rangle$ at $\sigma=0 \min ^{-1 / 2}$, that is

1032 constant for different values of $c_{L}$ (inset). The mean front speed for each value of $c_{L}$ and $\sigma$ was

1033 calculated by integrating equation (Fehler! Verweisquelle konnte nicht gefunden werden.)

1034 along 150 different landscapes with identical $\mathrm{c}_{\mathrm{L}}$ and fitting the mean front position versus time in 1035 the asymptotic propagation regime. 

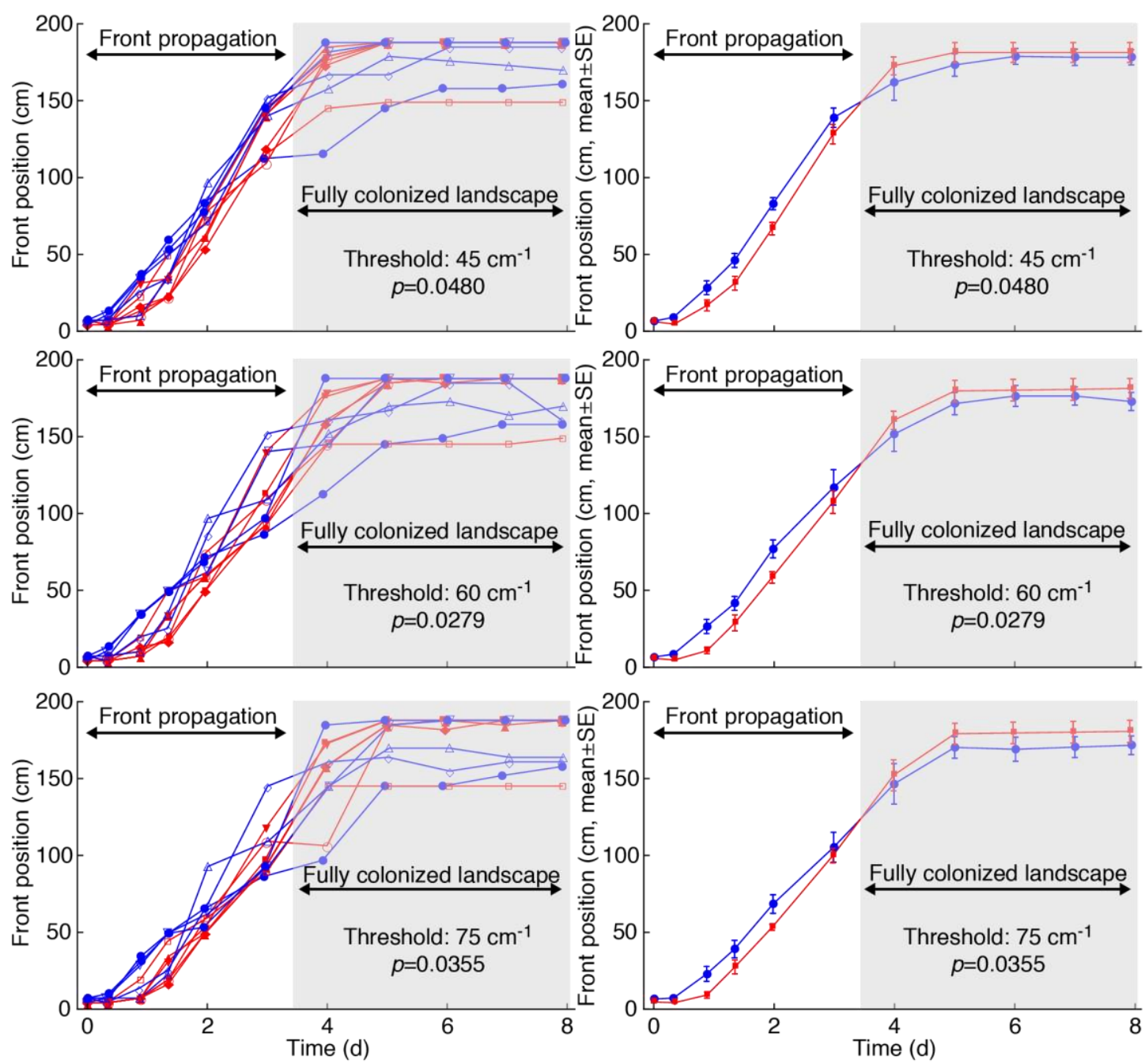

1037 Figure 9: Experimental spread in autocorrelated landscapes. Left: position of the front in each experimental replicate, identified by different symbols. Red and blue lines and symbols refer to replicates with identical large (red) or small (blue) resource autocorrelation length. Right: mean $( \pm \mathrm{SE})$ position of the front, calculated among replicates with identical large (red) or small (blue) resource autocorrelation length. Different rows refer to different threshold density values used to identify the position of the front. The gray shaded regions identify data points collected when at least one replicate had colonized the whole landscape. To avoid border effects, we excluded such points from the statistical analysis. In fact, at least one replicate with small autocorrelation length had reached the end of the landscape at time $\mathrm{t}=4 \mathrm{~d}$, and might have spread even further in a longer landscape. The reported p-values show that the autocorrelation treatment had a significant effect on the front propagation regardless of the choice of density threshold. 

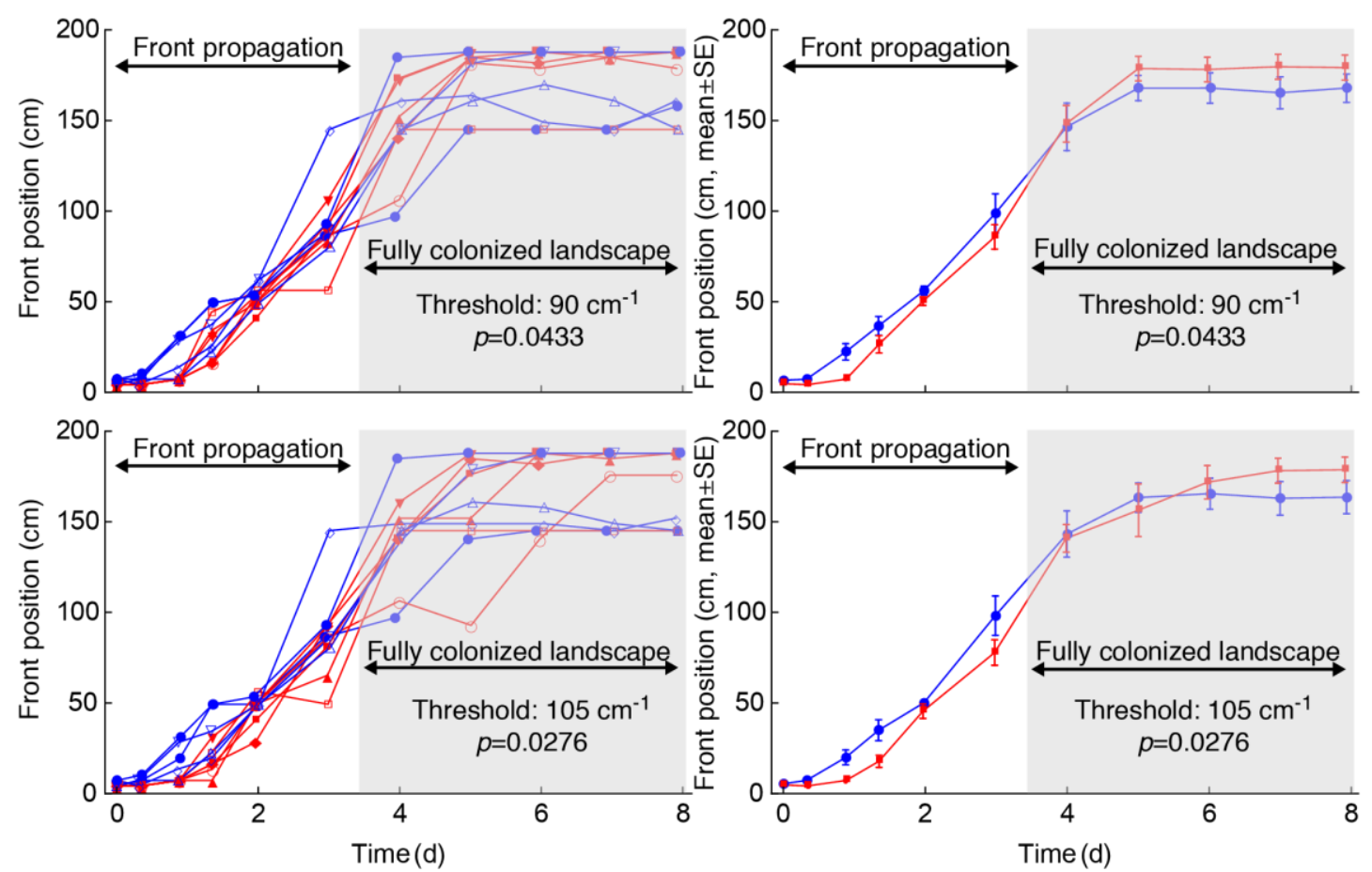

1049 Figure 10: Experimental spread in autocorrelated landscapes. Left: position of the front in each experimental replicate, identified by different symbols. Red and blue lines and symbols refer to replicates with identical large (red) or small (blue) resource autocorrelation length. Right: mean $( \pm \mathrm{SE})$ position of the front, calculated among replicates with identical large (red) or small (blue) resource autocorrelation length. Different rows refer to different threshold density values used to identify the position of the front. The gray shaded regions identify data points collected when at least one replicate had colonized the whole landscape. To avoid border effects, we excluded such points from the statistical analysis. In fact, at least one replicate with small autocorrelation length had reached the end of the landscape at time $\mathrm{t}=4 \mathrm{~d}$, and might have spread even further in a longer landscape. The reported p-values show that the autocorrelation treatment had a significant effect on the front propagation regardless of the choice of density threshold. 
1062 Bonachela, J. et al. 2012. Patchiness and demographic noise in three ecological examples. - J.

$1063 \quad$ Stat. Phys. 148: 723-739.

1064 Dewhirst, S. and Lutscher, F. 2009. Dispersal in heterogeneous habitats: thresholds, spatial scales, 1065 and approximate rates of spread. - Ecology 90(5): 1338-1345.

1066 Doering, C. R. et al. 2005. A numerical method for some stochastic differential equations with 1067 multiplicative noise. - Physics Letters A 344: 149-155.

1068 Dornic, I. et al. 2005. Integration of Langevin equations with multiplicative noise and the viability 1069 of field theories for absorbing phase transitions. - Phys. Rev. Lett. 94: 100601.

1070 Gillespie, D. T. 2000. The chemical Langevin equation. - The Journal of Chemical Physics 113: 1071 297-306.

1072 Giometto, A. et al. 2014. Emerging predictable features of replicated biological invasion fronts. 1073 Proc. Natl. Acad. Sci. U.S.A. 111: 297-301.

1074 Giometto, A. et al. 2015. Generalized receptor law governs phototaxis in the phytoplankton 1075 Euglena gracilis. - Proc. Natl. Acad. Sci. U.S.A. 112: 7045-7050. 